\title{
Neuroprotective effect of osmotin against ethanol-induced apoptotic neurodegeneration in the developing rat brain
}

\author{
MI Naseer ${ }^{1,2,5}$, I Ullah ${ }^{1,5}$, ML Narasimhan ${ }^{3}$, HY Lee ${ }^{1}$, RA Bressan ${ }^{3,4}$, GH Yoon ${ }^{1}$, DJ Yun ${ }^{4}$ and MO Kim ${ }^{\star, 1}$
}

Fetal alcohol syndrome is a neurological and developmental disorder caused by exposure of developing brain to ethanol. Administration of osmotin to rat pups reduced ethanol-induced apoptosis in cortical and hippocampal neurons. Osmotin, a plant protein, mitigated the ethanol-induced increases in cytochrome $c$, cleaved caspase-3, and PARP-1. Osmotin and ethanol reduced ethanol neurotoxicity both in vivo and in vitro by reducing the protein levels of cleaved caspase-3, intracellular $\left[\mathrm{Ca}^{2+}\right]_{\mathrm{cyt}}$, and mitochondrial transmembrane potential collapse, and also upregulated antiapoptotic Bcl-2 protein. Osmotin is a homolog of adiponectin, and it controls energy metabolism via phosphorylation. Adiponectin can protect hippocampal neurons against ethanol-induced apoptosis. Abrogation of signaling via receptors AdipoR1 or AdipoR2, by transfection with siRNAs, reduced the ability of osmotin and adiponectin to protect neurons against ethanol-induced neurodegeneration. Metformin, an activator of AMPK (adenosine monophosphate-activated protein kinase), increased whereas Compound C, an inhibitor of AMPK pathway, reduced the ability of osmotin and adiponectin to protect against ethanol-induced apoptosis. Osmotin exerted its neuroprotection via Bcl-2 family proteins and activation of AMPK signaling pathway. Modulation of AMPK pathways by osmotin, adiponectin, and metformin hold promise as a preventive therapy for fetal alcohol syndrome.

Cell Death and Disease (2014) 5, e1150; doi:10.1038/cddis.2014.53; published online 27 March 2014

Subject Category: Neuroscience

Maternal alcohol consumption exposes fetal brains to ethanol that disrupts brain development in humans and in animal models. ${ }^{1}$ The resulting neurological disturbances and developmental defects are manifested over the entire lifetime of the offspring and are referred to as fetal alcohol effects or fetal alcohol syndrome (FAS), depending on their severity. In humans, the effects of fetal brain alcohol exposure include craniofacial malformations as well as an array of neurological disorders including hyperactivity, learning and memory deficits, mental retardation, psychosis, depression, and schizophrenia. ${ }^{2,3}$ Presently, there are no effective treatments to prevent or reverse FAS after ethanol exposure. ${ }^{4}$

Studies of alcohol exposure in developmental animal models have provided insight into the possible mechanisms of ethanol-induced neurotoxicity and also permitted the study of interventions that mitigate the effects of ethanol. Such studies have established that ethanol exposure is associated with massive reduction in the number of neurons in the cerebral cortex, hippocampus, cerebellum, and olfactory bulb. ${ }^{5}$ Current studies provide strong evidence that neurodegeneration at substantial scale is the mechanism by which ethanol disrupts brain development in the rodent and, similarly, ethanol exposure disrupts development of the human fetal brain. ${ }^{6-8}$ Several pathways appear to contribute to the induction of neuronal death by ethanol. These include oxidative stress, rapid changes in intracellular $\mathrm{Ca}^{2+}$, excitotoxicity caused by the NMDA antagonist and GABA mimetic properties of ethanol, disruption of cell-cell interactions, and interference with the activity of growth factors. ${ }^{8-10}$ Regardless of the trigger, ethanol-induced apoptosis is associated with increased mitochondrial permeability and impaired mitochondrial function.

Several recent reports on the molecular mechanisms of ethanol action in brains suggest a connection with the mammalian hormone adiponectin. Adiponectin is a $30-\mathrm{kD}$ protein secreted by adipocytes whose serum level is positively correlated with insulin sensitivity, glucose and fatty acid use, cardiovascular protection, and protection against hypertension. ${ }^{11}$ At a mechanistic level, these metabolic effects are mediated by adiponectin largely via its effects on energy metabolism. ${ }^{12,13}$ Briefly, the interaction of adiponectin with its plasma membrane receptors AdipoR1 and AdipoR2 induces

\footnotetext{
${ }^{1}$ Department of Biology, College of Natural Sciences (RINS) and Applied Life Science (BK21 Plus), Gyeongsang National University, Jinju 660-701, Republic of Korea; ${ }^{2}$ Center of Excellence in Genomic Medicine and Research (CEGMR) King Abdulaziz University, PO Box 80216, Jeddah 21589, Saudi Arabia; ${ }^{3}$ Department of Horticulture and Landscape Architecture, Purdue University, West Lafayette, IN 47907-2010, USA and ${ }^{4}$ Department of Biochemistry and Applied Life Science (BK21 Plus), Gyeongsang National University, Jinju 660-701, Republic of Korea

*Corresponding author: MO Kim, Division of Life Science, College of Natural Sciences, Gyeongsang National University, Jinju 660-701, Republic of Korea. Tel: + 8255772 1345; Fax: + 8255772 1349; E-mail: mokim@gsnu.ac.kr

${ }^{5}$ These authors contributed equally to this study.

Keywords: osmotin; adiponectin; ethanol; neuroprotection; FAS

Abbreviations: FAS, fetal alcohol syndrome; AdipoR, adiponectin receptor; AMPK, adenosine monophosphate-activated protein kinase; P7, postnatal day 7; GD, gestational day; DMEM, Dulbecco's modified Eagle's medium; FJB, Fluoro-Jade B; PI, propidium iodide; PARP-1, poly [ADP-ribose] polymerase 1; TUNEL, terminal deoxynucleotidyl transferase dUTP nick-end labeling; PVDF, polyvinylidene difluoride; FBS, fetal bovine serum; MTT, 3-[4,5-dimethylthiazol-2-yl]-2,5-diphenly tetrazolium bromide
}

Received 25.9.13; revised 25.12.13; accepted 13.1.14; Edited by A Verkhratsky 
phosphorylation and activation of adenosine monophosphateactivated protein kinase (AMPK), a protein that senses depletion of cellular energy.

Adiponectin enters cerebrospinal fluid from blood. ${ }^{14}$ Expression of adiponectin receptor 1 (AdipoR1) is ubiquitous in rat brain, whereas the limited expression of AdipoR2 was observed in specific brain areas such as hypothalamus, cortex, and hippocampus. ${ }^{15}$ Administration of adiponectin by intracerebroventricular injection protects mouse hippocampal neurons against kainic acid-induced cell death, probably by improving vascular endothelial function and preserving the integrity of the blood brain barrier. ${ }^{16}$ Adiponectin protects against cerebral ischemic injury. ${ }^{17,18}$ Together, these data suggest that adiponectin has a widespread role in brain metabolism. The limited evidence available suggests that the mechanism of adiponectin action in brain tissue is likely to be the same as in other tissues. In arcuate hypothalamus of mice, adiponectin activates AdipoR1/R2 and subsequently induces phosphorylation of AMPK to systemically balance energy intake and expenditure. ${ }^{14}$ Administration of adiponectin by intracerebroventricular injection induces AMPK phosphorylation in rat hypothalamus. ${ }^{15}$ Thus, the mechanisms of adiponectin action in brain and other organs share similarities. In summary, adiponectin and adiponectin receptors exist in areas of brain that undergo the alcohol-induced apoptosis that is responsible for FAS. Therefore, we examined the neuroprotective effects of adiponectin-activated pathways against FAS. For reasons of economy, this study was conducted with a homolog of adiponectin that can be isolated and purified in large quantities from plant tissue. The adiponectin homolog used was a tobacco protein called osmotin.

We have shown here that osmotin protects rat fetal hippocampal neurons in vitro as well as neonatal hippocampal and cortical neurons in vivo against ethanol-induced apoptosis. Adiponectin also protected against ethanol insult in cultured rat fetal hippocampal neurons. Neuroprotection by osmotin and adiponectin was mediated by adiponectin receptors and AMPK activity. Together, our results suggest that the devastating effects of FAS can be prevented or reversed by therapeutic interventions that result in activation of AMPK, which may include the widely used antidiabetic drug metformin as well as adiponectin and osmotin. This study also demonstrates the value of osmotin as an economical experimental alternative to adiponectin. Osmotin-like proteins are ubiquitous in plants and occur in edible fruits, vegetables, and cereals. ${ }^{19}$ Therefore, our results also suggest that osmotin-like plant proteins could be a valuable source of neuroprotective agents against FAS.

\section{Results}

Osmotin protects against ethanol-induced apoptotic neurodegeneration in primary cultures of hippocampal neurons. Ethanol-induced craniofacial malformations involve apoptosis and neurodegeneration that occur within $12 \mathrm{~h}$ of exposure. ${ }^{7,8}$ A preliminary examination of the effect of osmotin on ethanol-induced neurodegeneration was studied in primary cultures of fetal rat brain hippocampal neurons by using double staining with Fluoro-Jade B (FJB) and propidium iodide $(\mathrm{PI})$. Upon treatment with ethanol for $24 \mathrm{~h}$, the neurons were markedly condensed and showed intense FJB and PI stains as compared with the control groups (Figure 1A). The ethanol plus osmotin group had an intermediate staining pattern, and quantitative measurements confirmed that there were fewer degenerating cells in the osmotin plus ethanol-treated group as compared with the cells exposed to ethanol alone and that neurodegeneration was reversed by the treatment of osmotin. Colocalization of FJB and PI stains in all samples suggested that ethanolinduced degeneration was closely associated with cell death.

The neuroprotective effect of osmotin was further quantified by the MTT (3-[4,5-dimethylthiazol-2-yl]-2,5-diphenly tetrazolium bromide) assay using primary cultures of hippocampal neuron exposed to ethanol for 12 and $24 \mathrm{~h}$. As shown in Figure 1B, supplementation ethanol in culture reduced cell viability to $55-60 \%$ as compared with the control group. However, osmotin along with the combination of ethanol improved cell viability significantly, up to $80-90 \%$ as compared with ethanol-treated groups (Figure 1B). Ethanol has NMDA antagonist and GABA mimetic properties, and the abnormal inhibition of neuronal activity by ethanol is believed to trigger apoptotic neurodegeneration. ${ }^{20}$ Agents that contribute to neuronal excitotoxicity or apoptosis disrupt calcium homeostasis by elevating the level of intracellular $\mathrm{Ca}^{+2} .21-23$ Previously, it has also been suggested that agents that inhibit the increase in intracellular $\mathrm{Ca}^{2+}$ have a neuroprotective effect. $^{21,22,24}$ It was therefore important to determine the effects of osmotin, ethanol, and ethanol plus osmotin exposure on the intracellular $\mathrm{Ca}^{2+}$ level. We observed that intracellular $\mathrm{Ca}^{2+}$ concentration was significantly elevated in cells exposed to ethanol $(100 \mathrm{mM})$ for $24 \mathrm{~h}$ compared with control groups (Figure 1C). Osmotin reversed the effect of ethanol on intracellular $\mathrm{Ca}^{2+}$ level in a concentrationdependent manner such that normal levels of intracellular $\mathrm{Ca}^{2+}$ could be restored by an appropriate osmotin dose $(0.16 \mu \mathrm{M})$.

Oxidative damage to mitochondria and mitochondrial dysfunction accompany ethanol-induced neurodegeneration and apoptosis. ${ }^{24}$ The mitochondrial membrane potential was therefore measured in primary hippocampal neuron cultures exposed to ethanol, osmotin, or osmotin plus ethanol for $24 \mathrm{~h}$ to investigate the mechanism by which cell viability is restored by osmotin treatment. As shown in Figure 2, treatment of neurons with ethanol resulted in a significant subpopulation of cells with low mitochondrial membrane potential (FL1 cells, $45.14 \%$ ). A smaller percentage of cells in the control, osmotintreated, and osmotin plus ethanol-treated groups had comparably low mitochondrial membrane potential $(29.29 \%$, $31.47 \%$, and $33.01 \%$, respectively), showing that ethanol induces the collapse of mitochondrial membrane potential and osmotin has a protective effect against ethanol-induced mitochondrial damage.

The second major alteration in mitochondria during apoptosis is an increase in the permeability of the mitochondrial outer membrane. ${ }^{25,26}$ Furthermore, we observed the change in protein expression upon ethanol exposure for $24 \mathrm{~h}$ in primary cultures of hippocampal neurons by using western blot analysis. Our results showed that ethanol-induced cell death was accompanied by increased accumulation of the proapoptotic protein Bax and a small but significant reduction 
A
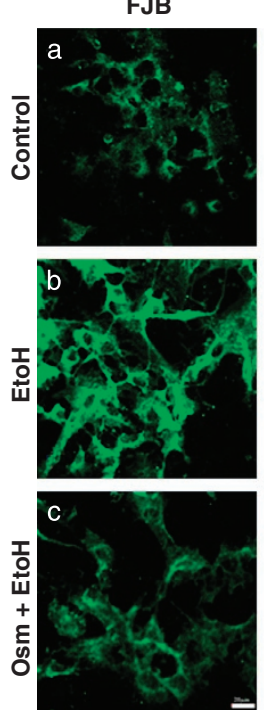

B
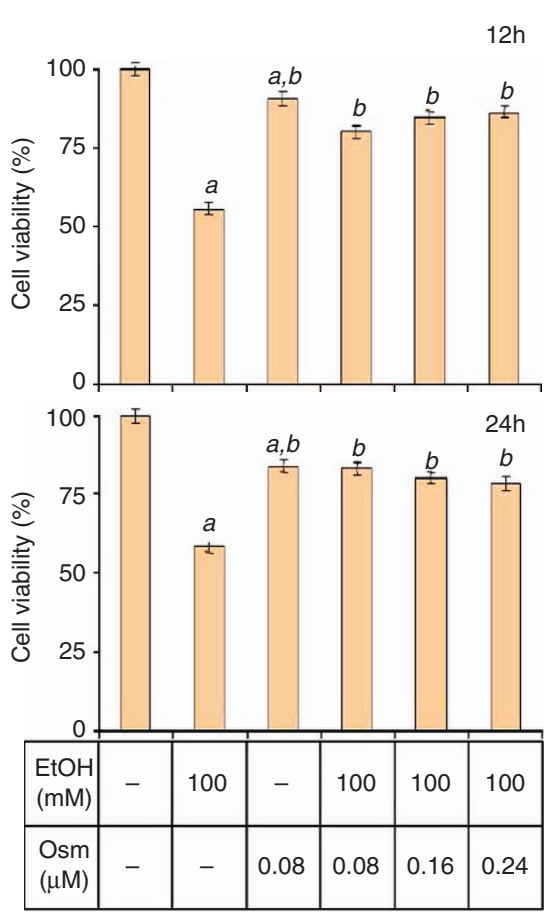

PI
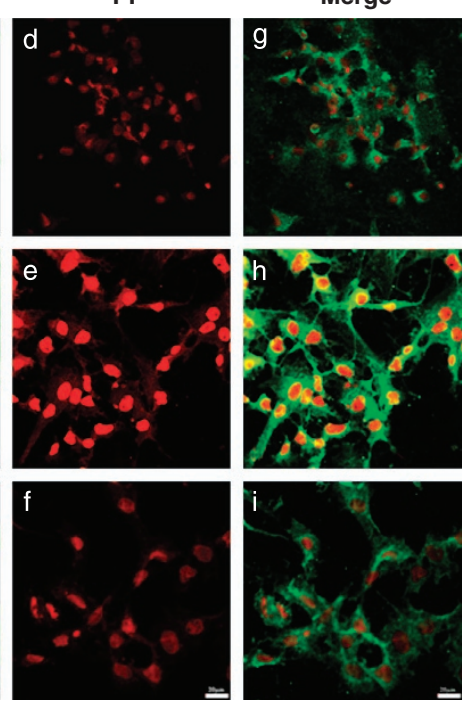

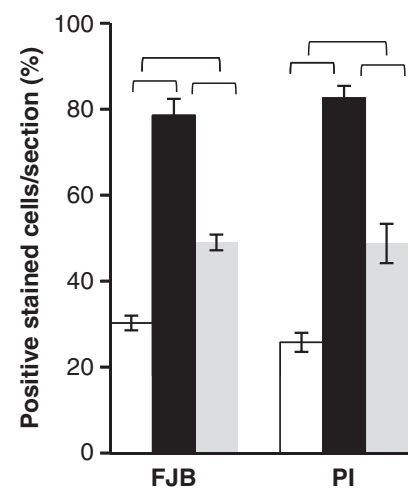

C
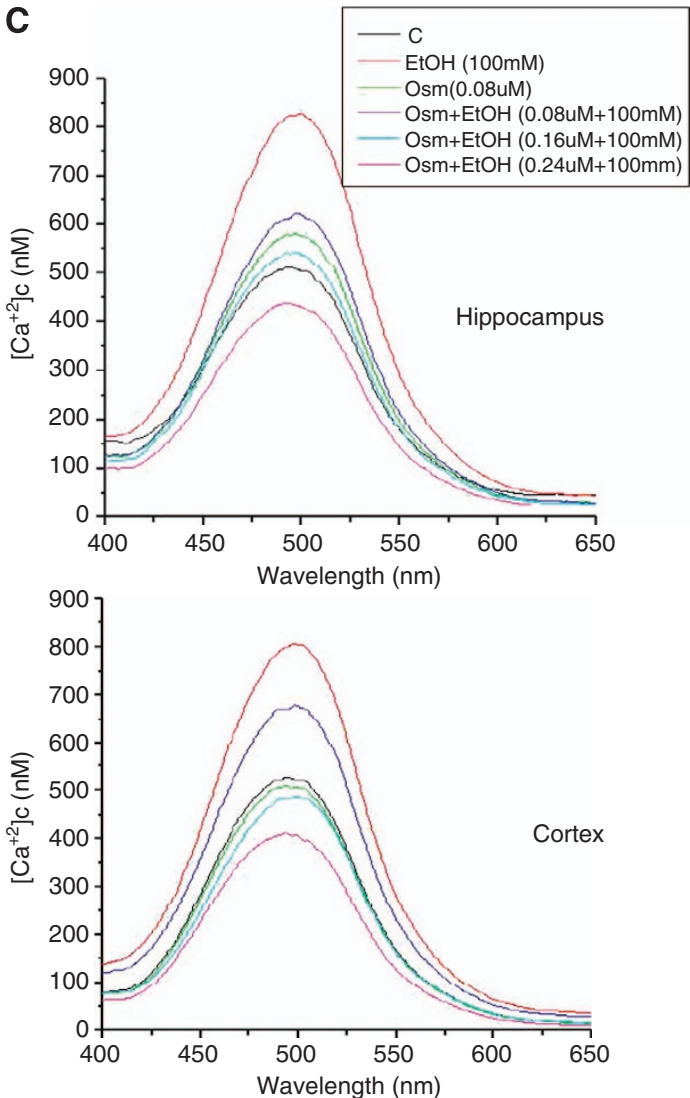

Figure 1 Osmotin protects against ethanol toxicity to primary cultures of fetal neurons. (A) Fluorescence analysis of neurodegeneration in primary cultures of fetal rat brain hippocampal neurons. Cultures were exposed to growth medium without (Control) and with ethanol (EtOH, $100 \mathrm{mM})$ or osmotin $(\mathrm{Osm}, 0.16 \mu \mathrm{M})$ plus EtOH $(100 \mathrm{mM})$ supplements for $24 \mathrm{~h}$ before staining with Fluoro-Jade B (FJB; green) and propidium iodide (PI; red). Magnification $\times 60$; scale bar, $20 \mu \mathrm{m}$. Shown in the graph are the percentages of FJB- and PI-positive cells per section $(n=4)$. Indicated pairs are significantly different at $P<0.05$. (B) MTT assay of cell viability in primary cultures of fetal rat brain hippocampal neurons. Cell viability was measured following exposure to ethanol and osmotin at the indicated concentrations for 12 and $24 \mathrm{~h}$. Data are the mean $\pm \mathrm{S}$.E. of three independent experiments $(n=3)$, with three plates in each experiment. Statistically significantly differences at $P<0.05$ are indicated by symbols. Symbols: $a$, different from control; $b$, different from ethanol $(100 \mathrm{mM})$. (C) Depicted are the analysis of cytosolic calcium concentrations in primary cultures of fetal rat brain hippocampal and cortical neurons exposed to the indicated treatments for $24 \mathrm{~h}$, followed by fura-2 AM labeling

in the level of the antiapoptotic protein Bcl-2 (Figures $3 a$ and b). The overall effect was a significant reduction in $\mathrm{Bcl}-2 / \mathrm{Bax}$ ratio that suggested that ethanol has a significant deleterious effect on mitochondrial membrane permeability. The most prominent effect of osmotin exposure was an increase in the level of the antiapoptotic protein $\mathrm{Bcl}-2$ compared with ethanol 

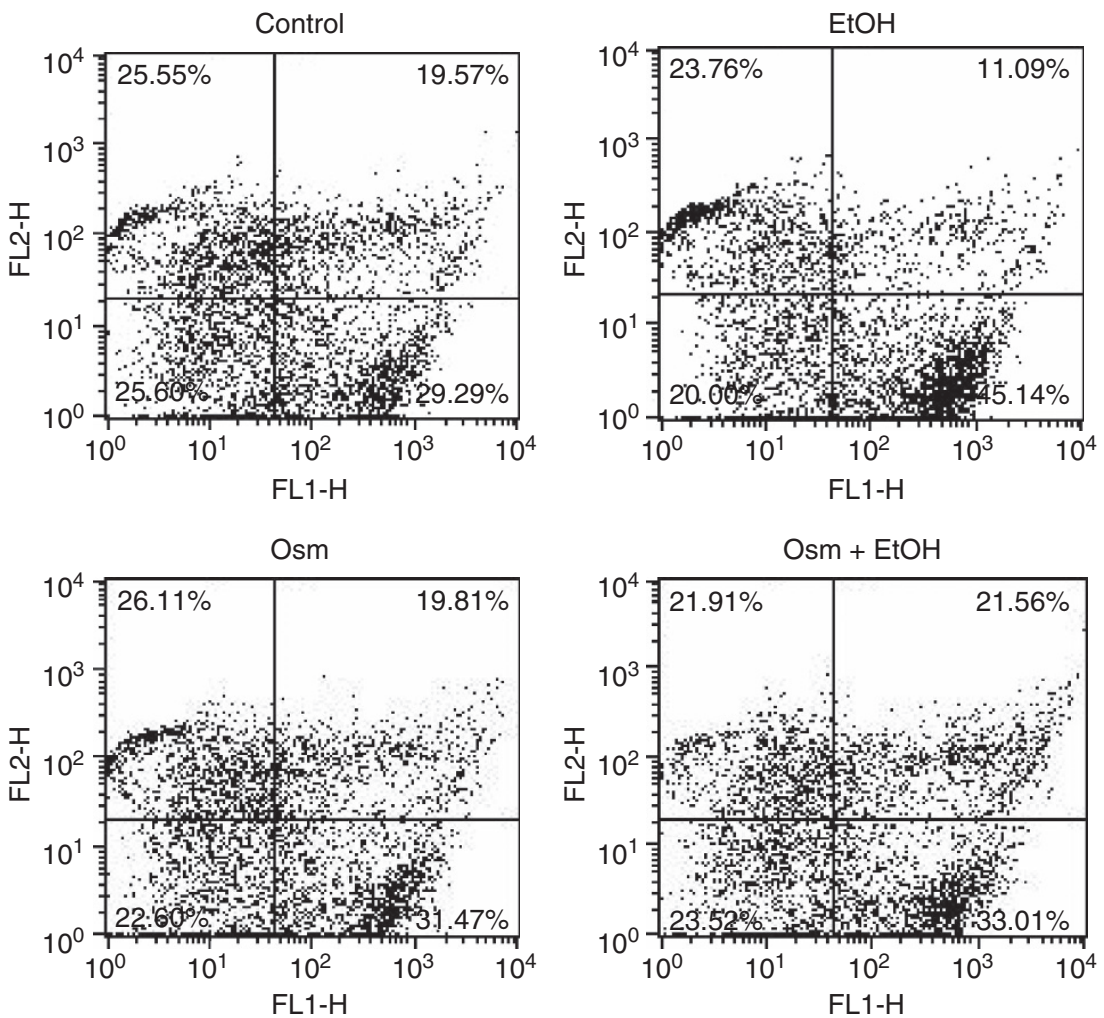

Figure 2 Osmotin protects against ethanol-induced collapse of mitochondrial membrane potential in primary cultures of fetal hippocampal neurons. Mitochondrial polarization was monitored by flow cytometric analysis of $\mathrm{JC}$-1-stained cells that were treated for $24 \mathrm{~h}$ with ethanol (EtOH, $100 \mathrm{mM}), 0 \mathrm{smotin}(\mathrm{Osm}, 0.16 \mu \mathrm{M})$, osmotin plus ethanol (Osm, $0.16 \mu \mathrm{M}+\mathrm{EtOH}, 100 \mathrm{mM}$ ), or untreated (Control). Collapse of mitochondrial membrane potential is associated with high FL1 fluorescence (green) and low FL2 fluorescence (red). The number in each quadrant indicates percent cell population in that quadrant out of total cells

treatment and almost no perturbation of the Bcl-2/Bax ratio compared with control group (Figure $3 b$ ). Cells exposed to osmotin plus ethanol had significantly higher Bcl-2/Bax ratios than ethanol-treated cells, suggesting that osmotin protects against ethanol-induced apoptosis by its protective effect on mitochondrial permeability.

Caspases are proteases that have a central role in the initiation and execution of apoptotic cell death. ${ }^{27}$ An increase in the level of cleaved caspase-3 was observed in ethanoltreated cells by western blot analysis, consistent with the notion that ethanol exposure induces apoptotic cell death in primary cultures of fetal hippocampal neurons (Figures $3 a$ and $b$ ). Osmotin treatment reduced the level of cleaved caspase-3. Because caspase- 3 can be activated by both mitochondrial and nonmitochondrial apoptosis pathways, this suggests that osmotin might have an antiapoptotic effect against a wide range of apoptotic stimuli. The level of cleaved caspase- 3 was lower in cells treated with osmotin plus ethanol compared with ethanol alone, confirming that osmotin has an antiapoptotic effect in ethanol-exposed cultured hippocampal neurons.

Osmotin inhibits ethanol-induced apoptosis in neonatal rat brain. To study the protective effect of osmotin against ethanol-induced apoptosis in vivo, P7 Sprague-Dawley rats were injected intraperitoneally with $20 \%$ ethanol in normal saline delivering $4 \mathrm{mg} / \mathrm{g}$ body weight. The pups were killed at different times after injection and apoptotic neurodegeneration was monitored as an increase in the level of cleaved caspase-3 in hippocampus (Supplementary Figure S1). Apoptotic neurodegeneration was manifested from $4 \mathrm{~h}$ up to at least $28 \mathrm{~h}$ after injection of ethanol. To establish an appropriate osmotin dose, pups were injected subcutaneously with various doses of osmotin in saline up to $30 \mathrm{~min}$ after ethanol injection and the expression level of cleaved caspase-3 was examined by western blotting $12 \mathrm{~h}$ later in hippocampal tissue (Figure 4A). Osmotin treatment reduced the cleaved caspase-3 level in ethanol-exposed tissues in a concentration-dependent manner. Significant reduction of cleaved caspase-3 level was observed at or above osmotin doses of $7 \mu \mathrm{g} / \mathrm{g}$ body weight. Based on these findings, ethanol was administrated at $4 \mathrm{mg} / \mathrm{g}$ body weight and osmotin at $15 \mu \mathrm{g} / \mathrm{g}$ body weight $30 \mathrm{~min}$ after ethanol treatment in the following experiments.

Apoptosis was observed in cortical and hippocampal tissues by TUNEL (terminal deoxynucleotidyl transferase dUTP nick-end labeling) staining after ethanol treatment and the extent of apoptosis was significantly reduced by osmotin post treatment (Figure 4B). Neurodegeneration was also visualized and quantified by FJB and PI staining. The anterior cingulate cortex and $\mathrm{CA} 1$ region of hippocampus that received ethanol had significantly greater numbers of FJB- and PIpositive cells compared with the control (Figures $4 C$ and D). In contrast, ethanol-treated rat pups that subsequently received osmotin showed a significant reduction in FJB- or PI-positive cells in these vulnerable brain regions as compared with ethanol treatment alone (Figure 4E). However, osmotin post 


\begin{tabular}{|c|c|c|c|c|c|}
\hline $\begin{array}{c}\text { EtOH } \\
(\mathrm{mM})\end{array}$ & - & 100 & - & \multicolumn{3}{|c|}{100} \\
\hline $\begin{array}{c}\text { Osm } \\
(\mu \mathrm{M})\end{array}$ & - & - & 0.08 & 0.12 & 0.16 \\
\hline
\end{tabular}

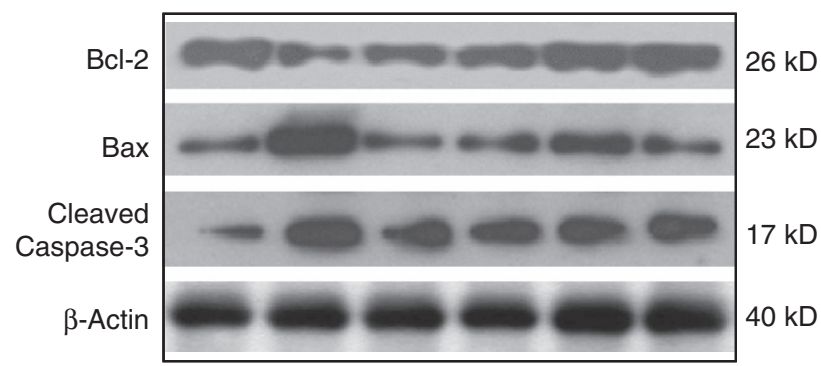

b
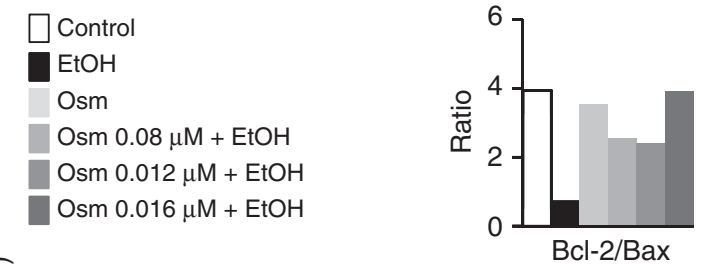

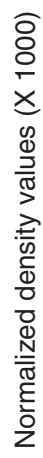

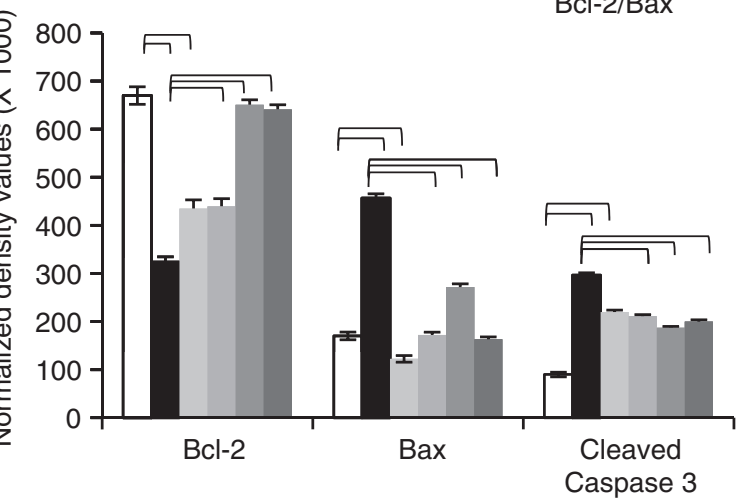

Figure 3 The protective action of osmotin against ethanol-induced apoptosis in primary cultures of fetal hippocampal neurons is evidenced by cellular levels of apoptotic marker proteins. (a) Cells were exposed for $24 \mathrm{~h}$ to normal growth medium containing no supplements (Control), or supplemented with ethanol (EtOH) and osmotin (Osm) as indicated. Shown are immunoblots of cell extracts that were fractionated by SDS-PAGE. The caspase-3 antibody used was capable of recognizing both pro- and cleaved caspase-3. However, only cleaved caspase-3 bands were visible in these samples. Blots were reacted with antibody to $\beta$-actin as loading control. (b) Shown is a quantitative analysis of the data in (a). Density values, normalized to actin signals, are expressed as mean \pm S.D. $(n=5)$ and are expressed as arbitrary units. Indicated pairs are significantly different at $P<0.05$. The inset shows the average $\mathrm{Bcl}-2$ to average Bax signal ratio across the treatments

treatment did not reduce the numbers of FJB- and PI-positive cells to the level found in the saline-treated control group, indicating that complete reversal of the neurodegenerative effect of ethanol was not achieved under our experimental conditions. Closer examination of PI-stained hippocampal tissue sections from brains of P7 pups revealed that in the hippocampal CA1 subfield, pyramidal neurons were scattered, stained more intensely with PI, and had different sizes and shapes in the ethanol-treated group compared with the osmotin and control groups (Figure 4F).

Mitochondrial dysfunction and apoptosis were further monitored using western blot analysis (Figure 5). Cytochrome c deficiency reduces cellular sensitivity to apoptotic stimuli that function through the mitochondrial pathway. ${ }^{28}$ This is because cytochrome $c$ is released from the intermembrane space of mitochondria into the cytosol in response to apoptotic stimuli that alter mitochondrial membrane potential or permeability that activates the caspase cascade. Our results (Figure 5 and Supplementary Figure S2a and b) show that ethanol-induced apoptotic neurodegeneration in hippocampal and cortical tissues of developing rat brains was characterized by significant downregulation of the ratio of antiapoptotic proteins (Bcl-2 and $\mathrm{Bcl}-\mathrm{XL}$ ) to proapoptotic proteins (Bax and Bak) and by significant upregulation of proapoptotic marker proteins such as cytochrome $c$, cleaved caspase- 9 , and cleaved caspase-3. Treatment with osmotin alone as well as osmotin post treatment of ethanol exposed to developing rat brains increased the ratio of antiapoptotic proteins (Bcl-2 and $\mathrm{Bcl}-\mathrm{XL}$ ) to proapoptotic proteins (Bax and Bak). Osmotin by itself affected some but not all of the tested downstream apoptotic markers. The levels of at least two of the three marker proteins, cytochrome $c$, cleaved caspase-9, and cleaved caspase-3, were significantly lower in the hippocampal and cortical tissues of pups that received osmotin after ethanol exposure compared with ethanol-treated group. Furthermore, both total and cleaved PARP-1 (poly [ADPribose] polymerase 1) levels were significantly reduced by osmotin alone or osmotin plus ethanol treatments compared with untreated and ethanol-treated groups, respectively. Osmotin and osmotin plus ethanol caused a decrease in total PARP-1 levels relative to untreated and ethanol-treated groups, respectively.

In addition, these brain tissues were histologically analyzed using immunofluorescence techniques for cytochrome $c$ and cleaved caspase-3. As depicted in the representative images (Figures $6 \mathrm{~A}$ and $\mathrm{B}$ ), consistent with results of western blot analysis (Figure 5 and Supplementary Figures S2a and b), ethanol injection increased the levels of cytochrome $c$ (FITClabeled, green) and caspase-3 (TRITC-labeled, red) in the CA1 region of hippocampus as well as the anterior cingulate cortex of developing rat brain. The decreased levels of cytochrome $c$ and caspase-3 in cortical tissues upon osmotin post treatment, compared with ethanol treatment alone (Figure 6B), was also consistent with our western blot results (Figure 5 and Supplementary Figures S2a and b). In contrast, osmotin post treatment significantly decreased only the level of cytochrome $c$ in total hippocampal tissue compared with ethanol treatment alone (Figure 5 and Supplementary Figures S2a and $b)$.

Overall, the molecular analyses support the imaging data in showing that ethanol administration to neonatal rat pups induced apoptotic neurodegeneration, whereas osmotin had a protective effect on mitochondria in neonatal rat brain tissues in vivo.

AMPK activity is involved in protecting against ethanolinduced neuronal apoptosis. Osmotin is a structural homolog of adiponectin. ${ }^{29}$ Adiponectin is present in cerebrospinal fluid and adiponectin receptors are expressed in most brain tissues. ${ }^{14,30,31}$ There are no reports on the involvement of adiponectin in protecting against ethanolinduced neuronal damage in developing brain tissues. It was 
therefore important to ascertain whether adiponectin mimics osmotin in protecting against ethanol neurotoxicity. Flow cytometric analysis of cell death by annexin V-PI staining in cultured hippocampal neurons treated with ethanol, osmotin, ethanol plus osmotin, or adiponectin was observed in this study. Our results showed that ethanol induced neurodegeneration, whereas adiponectin, like osmotin, protected against ethanol-induced cell death (Figures $7 a$ and $b$ ) that
A

\begin{tabular}{|c|c|c|c|c|c|c|}
\hline $\begin{array}{c}\text { EtOH } \\
(\mathrm{mg} / \mathrm{g} \text { b.w. })\end{array}$ & - & \multicolumn{5}{|c|}{4} \\
\hline $\begin{array}{c}\text { Osm } \\
(\mu \mathrm{g} / \mathrm{g} \text { b.w. })\end{array}$ & - & - & 1 & 7 & 15 & 30 \\
\hline
\end{tabular}

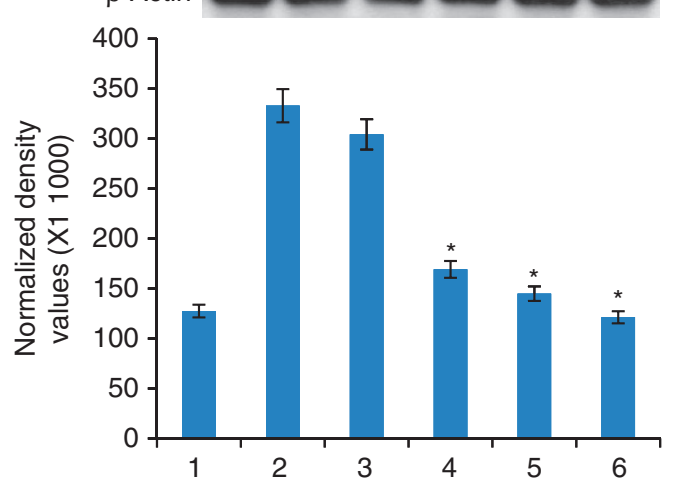

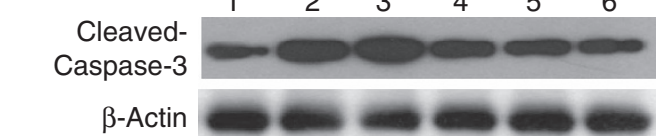

B
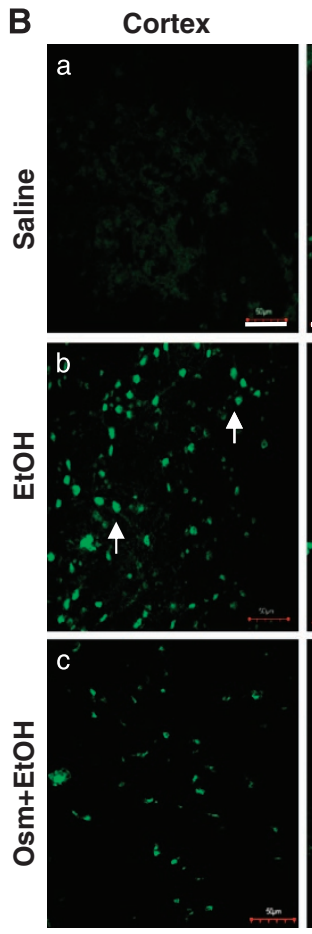

Hippocampus

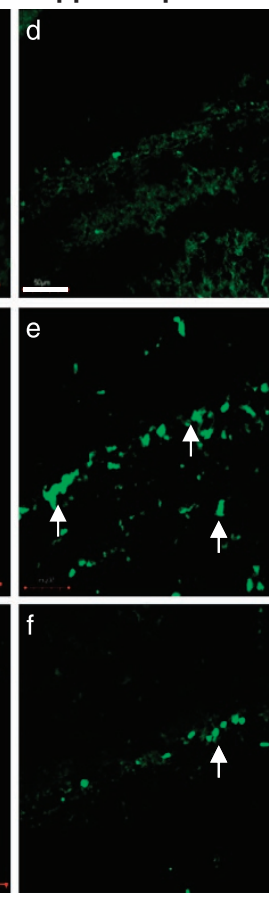

C

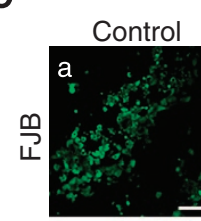

Hippocampus
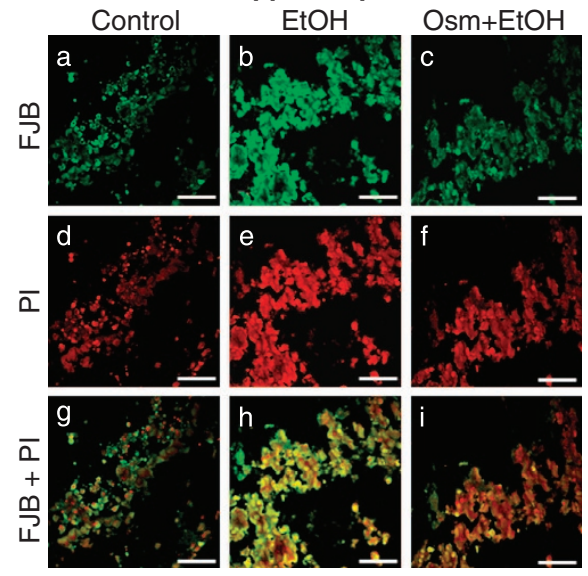

D

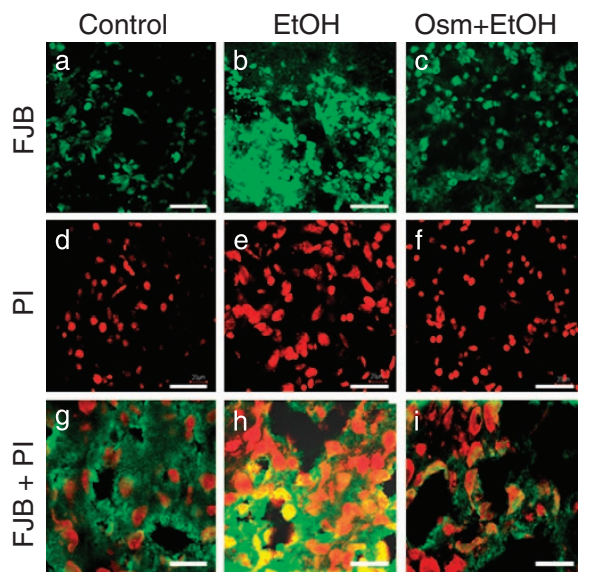

E
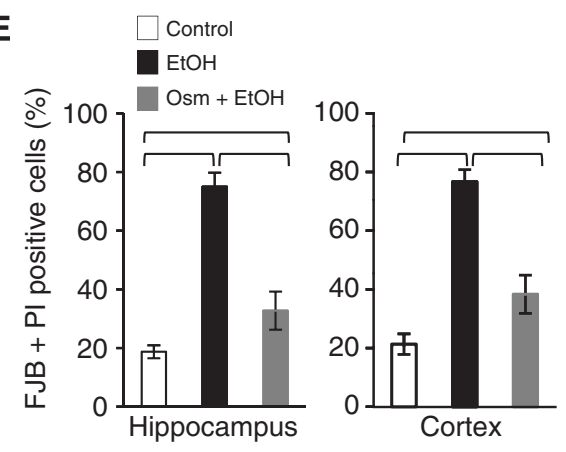

F

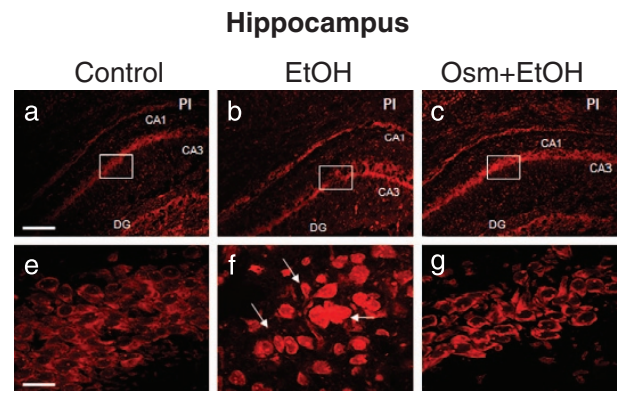


further confirm our results, as previously shown by Miele et al., ${ }^{32}$ that osmotin behaves like adiponectin as the structural and functional similarities are same between osmotin and human adiponectin. A similar conclusion was reached by observing that cleaved caspase- 3 in these cells was increased by ethanol treatment and that combining osmotin or adiponectin with ethanol partially reversed the effect of ethanol (Figure 7c).

Osmotin signaling via adiponectin receptors in murine C2C12 myotubes leads to the activation of AMPK. ${ }^{33}$ The activation of AMPK by $\mathrm{Thr}^{210}$ phosphorylation is a key downstream component of adiponectin signaling that mediates many of the beneficial effects of adiponectin. ${ }^{14,34,35} \mathrm{We}$ found that osmotin signaling in cultured hippocampal neurons also induced AMPK Thr $^{210}$ phosphorylation (Figure 7a). Furthermore, to test whether AMPK activation could have

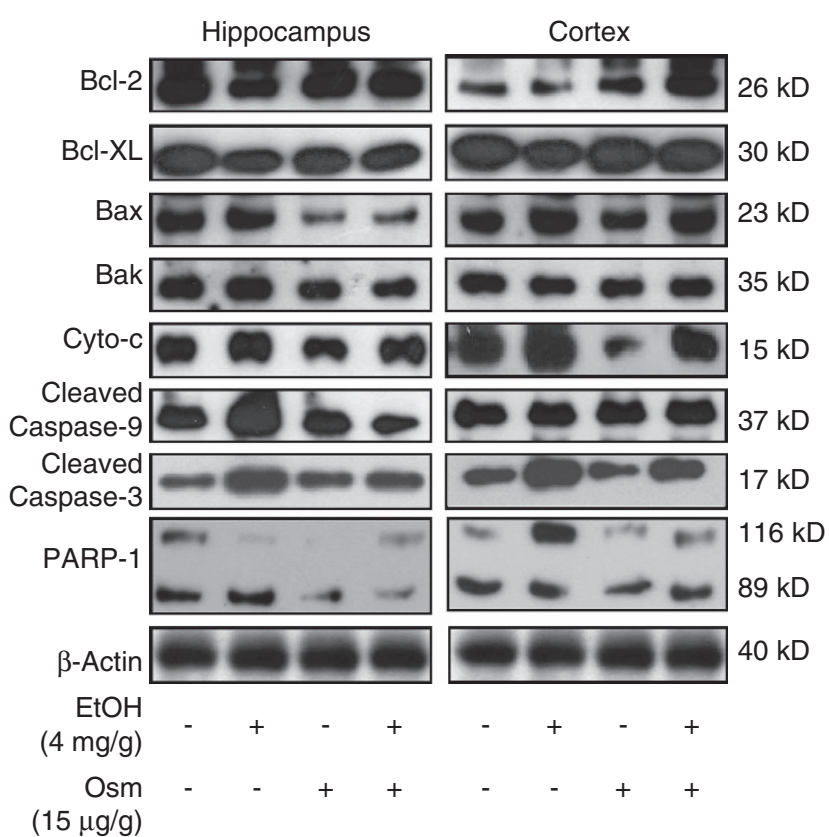

Figure 5 Levels of apoptotic marker proteins in developing rat brains suggest a protective action of osmotin in vivo against ethanol-induced apoptosis. $\mathrm{P} 7$ rat pups were injected with saline ( - ) or ethanol (EtOH; $4 \mathrm{mg} / \mathrm{g}$ b.w.) and again with osmotin (Osm; $15 \mu \mathrm{g} / \mathrm{g}$ b.w.) or saline $30 \mathrm{~min}$ later. Shown are immunoblots representing levels of the indicated apoptotic marker proteins in tissue extracts at $12 \mathrm{~h}$ after ethanol administration. $\beta$-Actin is taken as loading control in each case. The experiment was repeated three times and one representative experiment is shown. Quantification and statistical analysis of the complete data are shown in Supplementary Figures S2a and b a role in the protection afforded by osmotin and adiponectin against ethanol toxicity to fetal hippocampal neurons, we tested the effect of co-administration of Compound $\mathrm{C}$ along with osmotin, ethanol, and adiponectin with different combinations. Compound $\mathrm{C}$ is a widely used competitive inhibitor of AMPK activity. Co-administration of Compound C with osmotin and ethanol or adiponectin and ethanol partially reversed the protective action of osmotin and adiponectin on ethanol-induced cell death as measured by FACS analysis using annexin V-PI-stained cells (Figures 7a and b) and total caspase-3 protein expression level using western blot analysis (Figure 7c). There was a significant increase in the amount of cleaved caspase-3 after Compound $\mathrm{C}$ treatment, suggesting that inhibition of AMPK activity accelerated the progression of apoptosis.

Metformin is a widely used antidiabetic drug that activates AMPK indirectly via its action on an upstream kinase. ${ }^{36}$ To confirm that activation of AMPK can protect against ethanol-induced apoptosis, we also examined the effect of co-administration of metformin and ethanol. As shown in Figure 7, metformin reduced the toxic effect of ethanol as measured by annexin V-PI staining. Caspase-3 level upon metformin plus ethanol-treated cells was not significantly different from ethanol-treated group. It is possible that metformin was not as effective as osmotin or adiponectin because the metformin dose was not high enough or because the kinase targeted by metformin contributes only partially to osmotin and adiponectin signaling.

Having shown that the protective effect of osmotin requires activation of AMPK signaling pathway, we next tested whether adiponectin receptors were also required as shown in Figure 8a. Furthermore, cultured fetal hippocampal cells transfected with unrelated siRNA had higher levels of cleaved PARP-1 when exposed to ethanol, indicating apoptosis. Exposure to osmotin plus ethanol or adiponectin plus ethanol resulted in a significant reduction in cleaved caspase-3 and cleaved PARP-1 levels as compared with ethanol-treated group, suggesting that osmotin and adiponectin protected against ethanol toxicity. Cells transfected with a combination of siAdipoR1 and siAdipoR2, which had reduced expression of both AdipoR1 and AdopoR2 (Figures 8b and c), had higher levels of cleaved caspase- 3 and cleaved PARP-1 than cells transfected with unrelated siRNA, suggesting that basal signaling through these receptors had a prosurvival function. As expected, ethanol treatment elevated levels of cleaved caspase-3 and cleaved PARP-1 compared with the control group in cells transfected with a combination of siAdipoR1 and siAdipoR2, indicating that ethanol induced apoptosis.

Figure 4 Osmotin inhibits ethanol-induced apoptosis in vivo. P7 rat pups were injected with saline ( - ) or ethanol (EtOH; $4 \mathrm{mg} / \mathrm{g} \mathrm{b.w.)} \mathrm{and} \mathrm{again} \mathrm{with} \mathrm{osmotin} \mathrm{(Osm)} \mathrm{or}$ saline 30 min later. (A) The treatments are indicated above the blots. Level of cleaved caspase- 3 and $\beta$-actin were analyzed in hippocampal part of brain by western blot at $12 \mathrm{~h}$ after ethanol administration. The graph shows the quantification of cleaved caspase-3 levels by densitometry. The values were normalized to $\beta$-actin signals and represent mean \pm S.D. $(n=3)$. Significant difference from the ethanol group at $P<0.01$ is indicated by an asterisk. (B) Apoptotic DNA fragmentation in brain tissues was evaluated with TUNEL staining $12 \mathrm{~h}$ after ethanol administration. The osmotin dose was $15 \mu \mathrm{g} / \mathrm{g} \mathrm{b.w}$. The arrows indicate TUNEL-stained nuclei in neurons. Magnification $\times 40$; scale bar, $50 \mu \mathrm{m}$. (C) P7 rat pups were injected with saline (Control) or ethanol (EtOH; $4 \mathrm{mg} / \mathrm{g}$ b.w.) and again with osmotin (Osm; $15 \mu \mathrm{g} / \mathrm{g}$ b.w.) or saline $30 \mathrm{~min}$ later. Pups were killed at $12 \mathrm{~h}$ after ethanol injection for analysis of neurodegeneration by propidium iodide (PI) and Fluoro-Jade B (FJB) staining. (C) Shown is stained sections from the CA1 region of the hippocampus. Magnification $\times 60$; scale bar, $20 \mu \mathrm{m}$. (D) Shown are stained sections from the anterior cingulated cortex. (a-f) Magnification $\times 40$; scale bar, $20 \mu \mathrm{m}$; ( $\mathrm{g}-\mathrm{i})$ magnification $\times 100$; scale bar, $10 \mu \mathrm{m}$. (E) Quantification of the data represented in (A) and (B). Data represent means \pm S.D. ( $n=4$, hippocampus; $n=3$, cortex). Sample pairs that were significantly different at $P<0.05$ are indicated. $(\mathbf{F})$ Shown are views of the CA1, CA3, and DG regions of hippocampal after PI staining at low ( $\times 20$; scale $\mathrm{bar}=20 \mu \mathrm{m})$ and high $(\times 100$; scale bar $=10 \mu \mathrm{m})$ magnification. Rectangles indicate the CA1 regions that are shown in the magnified views. Arrows indicate shrunken and damaged neurons 
A
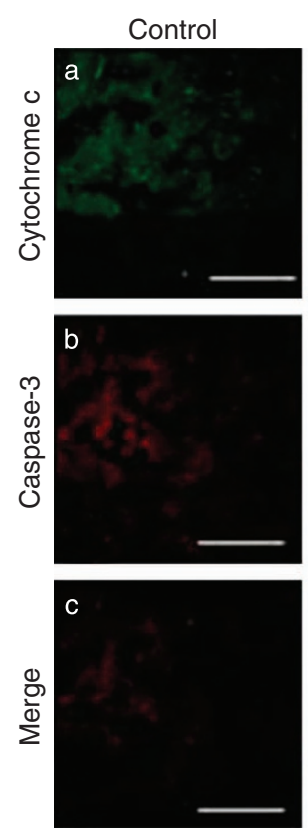

B
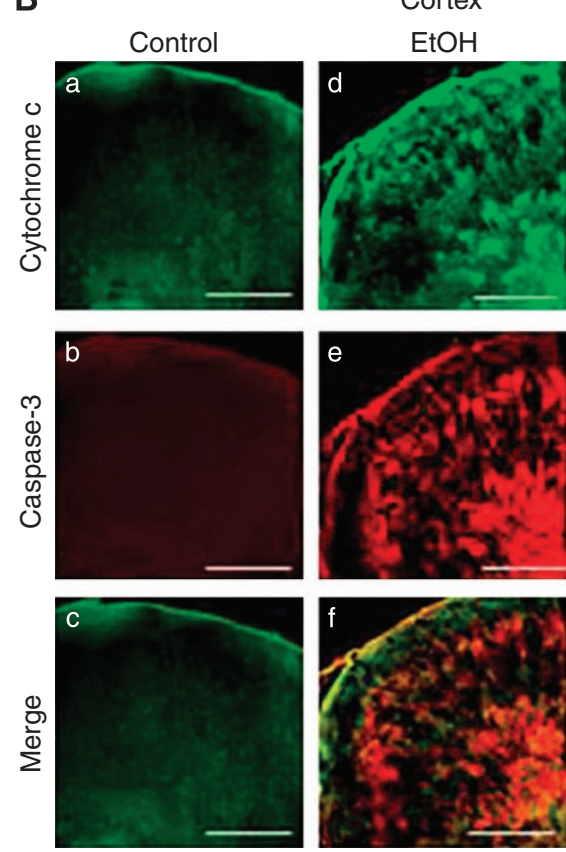

$\mathrm{OSM}+\mathrm{EtOH}$
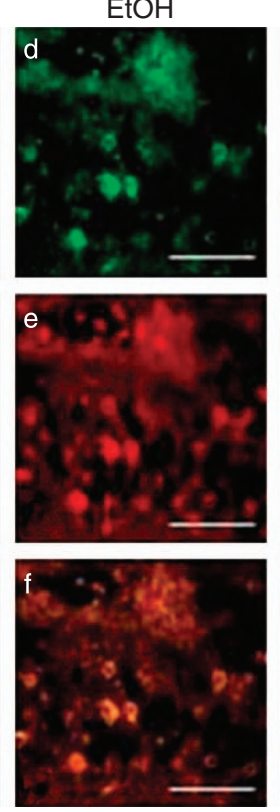

Cortex
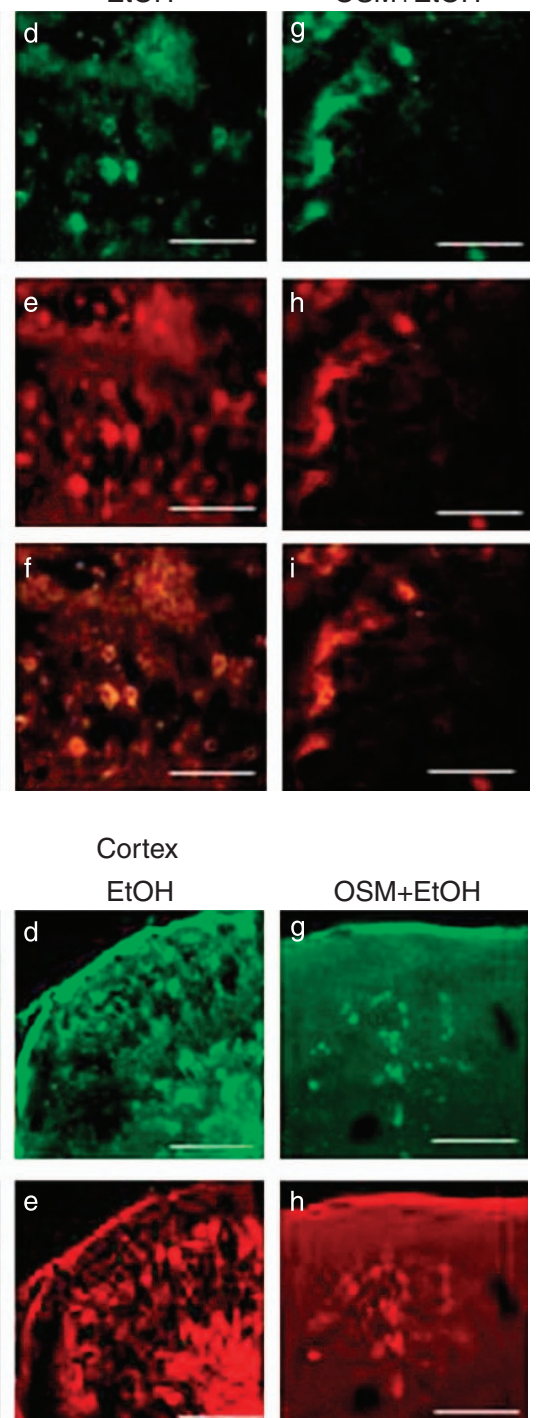

$\mathrm{OSM}+\mathrm{EtOH}$
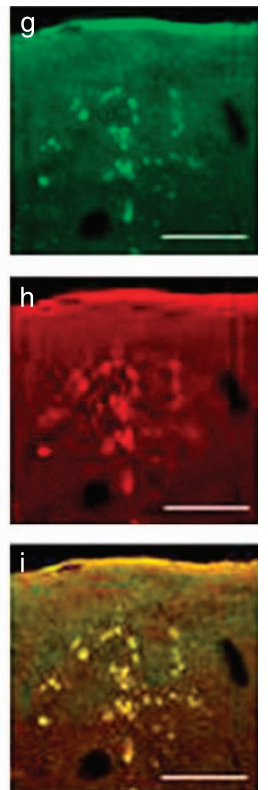

Figure 6 Immunofluorescence analysis of cytochrome $c$ and caspase-3 levels showing the in vivo neuroprotective action of osmotin against ethanol-induced apoptosis in developing rat brain. P7 rat pups were injected with saline (Control) or ethanol (EtOH; $4 \mathrm{mg} / \mathrm{g}$ b.w.) and again with osmotin (Osm; $15 \mu \mathrm{g} / \mathrm{g}$ b.w.) or saline $30 \mathrm{~min}$ later. Pups were killed at $12 \mathrm{~h}$ after ethanol injection for analysis. Shown are tissue sections from the CA1 region of the hippocampus $(\mathbf{A})$ and the anterior cingulate cortex (B) that were double stained for cytochrome $c$ (FITC-label, green) and caspase-3 (TRITC-label, red). Yellow color (merge) indicates colocalization. (a-c) magnification, $\times 20$; (d-i) magnification, $\times 40$; scale bar, $20 \mu \mathrm{m}$

However, in siAdipoR1- plus siAdipoR2-transfected cells, the levels of cleaved caspase-e and cleaved PARP-1 in osmotin plus ethanol or adiponectin plus ethanol-treated groups were higher than or equal to that of the ethanol-treated group

(Figures $8 \mathrm{~b}$ and $\mathrm{c}$ ) suggesting that osmotin and adiponectin protected against ethanol toxicity. Furthermore, we also studied the exposure of osmotin plus ethanol and adiponectin plus ethanol in both unrelated and AdipoR1 plus AdipoR2 siRNA-transfected cells, resulting in a significant increase in p-AMPK expression levels as compared with ethanol-treated group in unrelated siRNA and significant decrease in p-AMPK expression in siAdipoR1- plus siAdipoR2-transfected cells (Figure 8d). Therefore, it is concluded that osmotin showed its neuroprotective effect through adiponectin receptors that are required for the protective action of osmotin and adiponectin against ethanol-induced neurodegeneration in hippocampal neurons.

\section{Discussion}

In this study we have demonstrated that plant protein osmotin, an adiponectin homolog, protects against alcohol-induced neurodegeneration in developing rat brain. Co-administration of osmotin with ethanol to primary cultures of hippocampal neurons derived from prenatal rat brain protected against ethanol-induced apoptosis. The neuroprotective action of osmotin against ethanol-induced apoptosis in prenatal rat neurons resulted from its ability to function like adiponectin, and initiate intracellular signaling via adiponectin receptors. Osmotin post treatment by subcutaneous injection prevented ethanol-induced neuronal apoptosis in the anterior cingulate cortex and the CA1 hippocampal region of neonatal developing rat brains. Adiponectin indirectly protects hippocampal neurons against kainic acid-induced excitotoxicity by improving vascular endothelial function to prevent blood brain barrier leakage. ${ }^{16}$ Recently, it was proved that osmotin showed structural and functional similarity with human adiponectin. ${ }^{32}$

Observations made at the cellular level using FACS and immunofluorescence techniques and the tissue level using immunoblotting techniques showed that ethanol-induced apoptosis in the developing rat brain was associated with mitochondrial dysfunction (Figures 2, 3, 5, and 6), similar to its effect on the hippocampus of fetal guinea pig brain. ${ }^{37}$ The protective action of osmotin against ethanol-induced toxicity appears to function at the mitochondrial level (Figures 2, 3, 5, and 6).

The most significant and consistent effect of combined osmotin and ethanol administration was the increase in the levels of antiapoptotic family members (e.g., Bcl-2, Bcl-XL) to the proapoptotic family members (e.g., Bax, Bak) compared with ethanol treatment alone (Figures 3 and 5 and Supplementary Figures $\mathrm{S} 2 \mathrm{a}$ and $\mathrm{b}$ ). This suggests that the influence of osmotin on the levels of the Bcl-2 family proteins could be a significant component of its neuroprotective action. The antiapoptotic Bcl-2 family members are particularly important in preventing neuronal apoptosis and neurodegeneration. Their roles in maintaining mitochondrial outer membrane integrity and inefficient energy generation through the control of the organization of mitochondrial networks independent of their effect on mitochondrial outer membrane permeability contribute to their neuroprotective effect. ${ }^{38,39}$ Thus, regulation of cellular levels of $\mathrm{Bcl}-2$ family members may be a direct way to influence apoptosis. Interestingly, adiponectin protects against 1-methyl-4-phenylpyridinium ion 
a
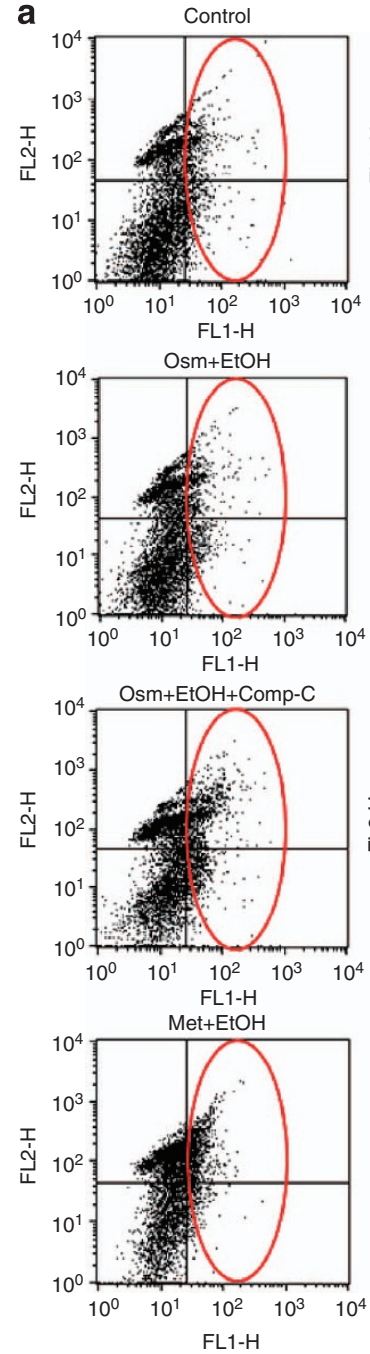
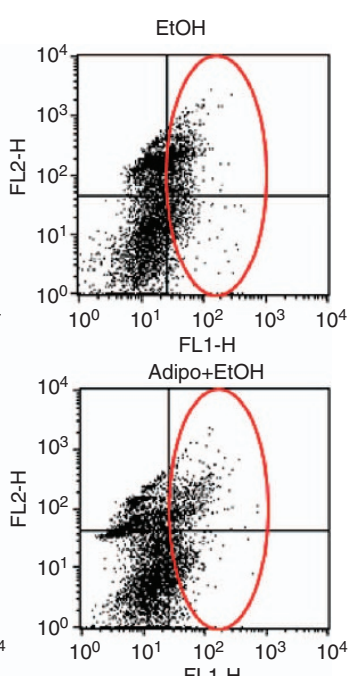

$10^{4}$ Adipo+EtOH+Comp-C
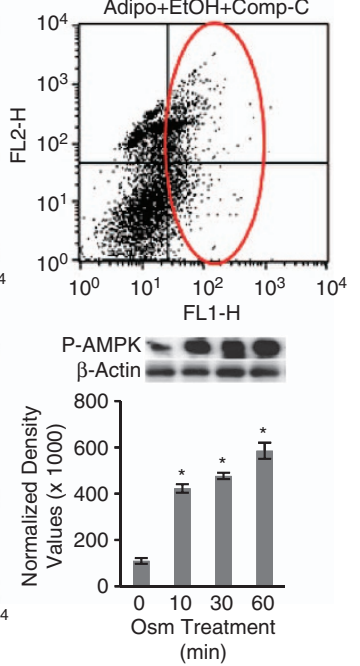

b
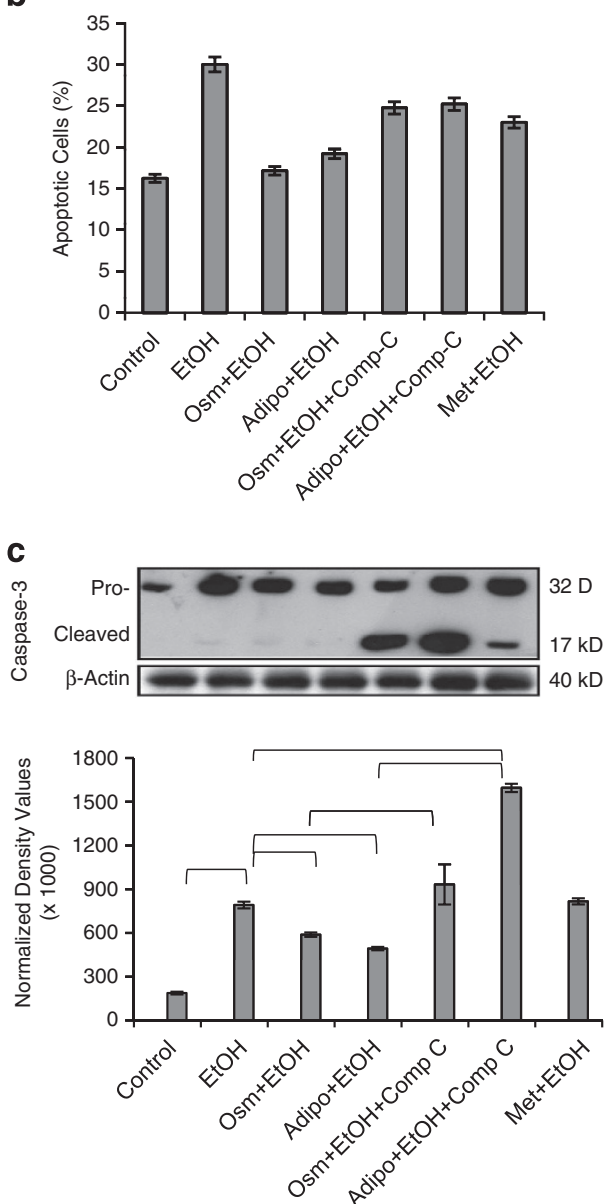

Figure 7 AMPK activity is involved in protection against ethanol-induced apoptosis. (a) Flow cytometric analysis of apoptotic cells by annexin V-propidium iodide staining. Primary cultures of fetal (GD 17.5) hippocampal neurons were treated with fresh growth medium without (Control) or with indicated combinations of ethanol (100 mM), osmotin $(0.16 \mu \mathrm{M})$, adiponectin $(0.1 \mu \mathrm{M})$, Compound $\mathrm{C}(20 \mu \mathrm{M})$, or metformin $(5 \mathrm{mM})$ supplements for $24 \mathrm{~h}$ and then analyzed. Cells positive for either Annexin V (early apoptotic) or both annexin $\mathrm{V}$ and propidium iodide (late apoptotic) were counted and they lie in the quadrants indicated by the red oval. Shown in the bottom right corner are the results of immunoblot analysis of phospho-AMPK (P-AMPK) levels in these cells at different times after osmotin $(0.16 \mu M)$ treatment. P-AMPK signals were normalized to $\beta$-actin signals and expressed as mean \pm S.D. $(n=5)$. Asterisk indicates significant difference from time zero group at $P<0.01$ (b) Shown are quantitative results of the flow cytometric analysis $(n=3)$, expressed as the percentages of the apoptotic cells in the population. (c) Shown are results of western blot analysis of cell extracts of the hippocampal neurons at the end of these treatments. Cell extracts were fractionated by SDS-PAGE and analyzed for caspase- 3 and $\beta$-actin by immunoblotting. Caspase-3 signals were normalized to $\beta$-actin signals and expressed as mean \pm S.E. $(n=5)$. Sample pairs that are significantly different from one another at $P<0.05$ are indicated

toxicity in neuroblastoma cells, probably by increasing antioxidant levels and affecting the $\mathrm{Bax} / \mathrm{Bcl}-2$ ratio. ${ }^{40}$ Experiments are required to elucidate the connection between osmotin and the cellular levels of the $\mathrm{Bcl}-2$ family proteins and its significance.

AMPK activation appears to be an important factor in the protective action of osmotin and adiponectin against ethanol neurotoxicity (Figure 7). Thus, metformin, an upstream activator of AMPK, also protected against ethanol neurotoxicity (Figure 7). Administration of metformin to diabetic rats prevents oxidative imbalance in brain. ${ }^{41}$ Metformin also protects against etoposide-induced damage in primary cultures of cortical neurons obtained from prenatal rat brains. In this model of diabetic neuropathy, prevention of mitochondrial dysfunction (collapse of mitochondrial membrane potential and release of cytochrome $c$ ) by metformin has been suggested as the basis for its neuroprotective effects. ${ }^{42}$ The relative contribution of the $\mathrm{Bcl}-2$ family proteins and AMPKdependent pathways to the protective action of osmotin toward mitochondria in developing neurons remains to be elucidated.

Osmotin belongs to a family of ubiquitous plant proteins termed PR-5 proteins. ${ }^{22}$ The best-studied member of this class is the commercially available sweetener, thaumatin. PR-5 proteins are quite abundant in fruits and vegetables such as ripe grapes, plantains, and bananas. ${ }^{43,44}$ This class of proteins therefore represents a rich source of potential adiponectin mimics that could be used for adiponectin structure-function studies or for the design of treatments for FAS. 
a

\begin{tabular}{|l|c|c|}
\hline & $\begin{array}{c}\text { Unrelated } \\
\text { siRNA }\end{array}$ & $\begin{array}{c}\text { AdipoR1+ } \\
\text { R2 siRNA }\end{array}$ \\
\hline AdipoR1 & & \\
\hline AdipoR2 & & \\
\hline GAPDH & & \\
\hline
\end{tabular}

b
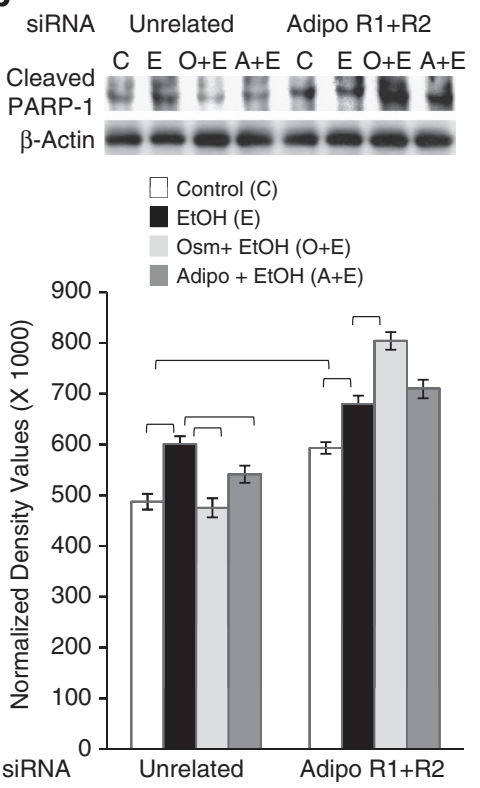

c

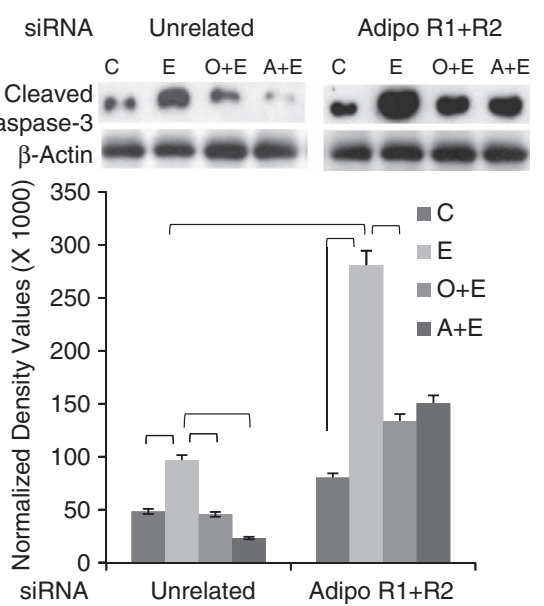

d

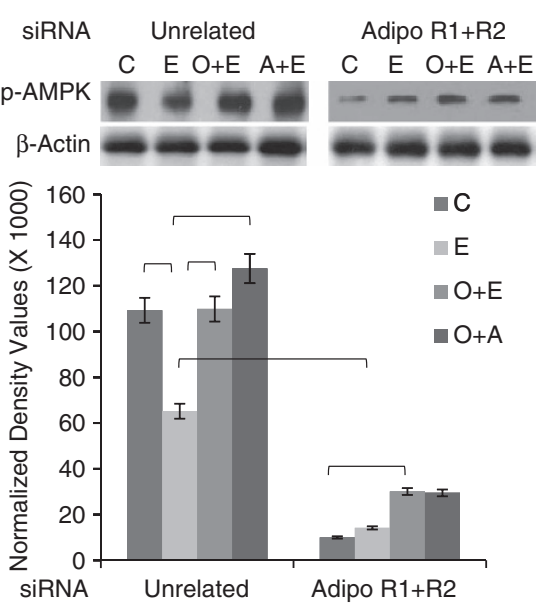

Figure 8 The protective effect of osmotin on ethanol-induced apoptosis requires adiponectin receptors. (a) Downregulation of adiponectin receptor expression by transfection with siRNA. Shown is the RT-PCR analysis of AdipoR1 and AdipoR2 mRNA expression in primary fetal (GD 17.5) hippocampal neurons that were transfected with unrelated siRNA or AdipoR1 plus AdipoR2 $(1: 1)$ siRNA. GAPDH mRNA levels are shown for normalizing expression levels. (b-d) Primary cultures of fetal hippocampal neurons that were transfected with a mixture (1:1) of AdipoR1 and AdipoR2 (AdipoR1 + R2) siRNAs and unrelated siRNA were treated with ethanol (EtOH; $100 \mathrm{mM}$ ), osmotin (Osm; $0.16 \mu \mathrm{M})$, or adiponectin (Adipo; $0.1 \mu \mathrm{M}$ ) in combinations for $24 \mathrm{~h}$. Cleaved PARP-1, cleaved capsase-3, and p-AMPK levels were then analyzed in extracts of the cultured hippocampal neurons by western blotting. Shown are the immunoblots for detection of cleaved PARP-1 (b), cleaved caspase-3 (c), p-AMPK (d), and $\beta$-actin as loading control in all cases. The graph represents normalized density values for the immunoblots signals expressed as mean \pm S.D. $(n=4)$. Sample pairs that are significantly different from one another at $P<0.05$ are indicated

Osmotin is a plant protein and these plant-derived components may be used for investigation of neurodegenerative disorders, obesity, and cancers, and play important role for the prevention of disease. Nutritional energy control or caloric restriction has been recommended to provide long life and reduce carcinogenesis. ${ }^{45}$ Several mechanisms such as growth factors, anabolic hormones, inflammatory cytokines, and oxidative stress markers linked to various malignancies have been anticipated for the beneficial effects and showed connection with molecules such as adiponectin and AMPK. ${ }^{46,47}$ In this study, we have shown that osmotin is a stable protein and adiponectin receptors in rat brain bind to osmotin as well as adiponectin. Osmotin a dietary compound showed functional similarity with adiponectin. ${ }^{29}$ Moreover, adiponectin plant-derived homolog, osmotin, showed beneficial effect against colitis. ${ }^{48}$

Osmotin, a plant protein, may play an important role to mimic the hormone that mitigates diabetes, obesity, cancer, and neurodegenerative disorders. This demonstrates the value of osmotin as an economical experimental alternative to adiponectin. Our results also suggested that osmotin could be a valuable source of neuroprotective agents against FAS and can be used to deliver health benefits. Using plant-derived adiponectin homologs, osmotin may become a therapeutic option against neurodegenerative disorders such as FAS.

\section{Materials and Methods}

Osmotin purification. Osmotin was purified from salt-adapted cultured tobacco cells as described previously. ${ }^{49}$ Endotoxin was removed using a prepacked ActiClean Etox column (Sterogene Bioseparations, Carlsbad, CA, USA). The final preparation was judged to be $>99 \%$ homogeneous by 2DGE and by LC-MS of peptide fragments obtained by trypsine digestion. The endotoxin content of osmotin was $<0.03 \mathrm{EU} / \mathrm{mg}$ protein.

Animal treatment. Female $(n=35)$ Sprague-Dawley rats $(250 \mathrm{~g}$, Gyeongsang National University, Neurobiology Laboratory, Chinju, South Korea) were housed in a temperature-controlled environment with lights from 0600 to $2000 \mathrm{~h}$ 
with food and water ad libitum. Animals were treated in accordance with standard guidelines for laboratory animal care. All efforts were made to minimize the number of animals used and their suffering. All the experimental procedures were approved by the local animal ethics committee of the Division of Applied Life Sciences, Department of Biology, Gyeongsang National University, South Korea. The animals were randomly divided into two experimental groups:

1. Timed pregnant (the day of insemination equals to gestational day (GD) 0.5 ). At GD 17.5, rats received an i.v. injection of pentobarbital sodium ( $3 \mathrm{mg} / 100 \mathrm{~g}$ body weight) and were then killed by decapitation.

2. Postnatal day 7 (P7) Sprague-Dawley pups were used in in vivo experiments and were equally distributed in four groups, that is, control, ethanol, osmotin, and osmotin plus ethanol. Ethanol (4 mg/g body weight) was delivered by subcutaneous injection. A saline solution of osmotin $(15 \mu \mathrm{g} / \mathrm{g})$ was delivered by subcutaneous injection at $30 \mathrm{~min}$ after ethanol injection. Saline injections of equal volume were used for the control group.

Primary cell culture and drug treatment. Primary cultures of hippocampal and cortical neurons were generated from hippocampus and cortex isolated from rat fetuses at GD 17.5 as described previously. ${ }^{50}$ After 4 days of growth, the growth medium was replaced with fresh medium without (control) or with supplements as indicated in the Figures. Cultures were analyzed $24 \mathrm{~h}$ later.

Western blotting. Western blot analysis was done as previously described with some modification. ${ }^{50}$ Briefly, primary cultured hippocampal cells and tissue from both hippocampal and cortical brain from $\mathrm{P} 7$ rat pups were homogenized in cell lysis buffer (Cell Signaling Technology, Danvers, MA, USA) and were supplemented with $100 \mathrm{mM}$ PMSF. Extracts were fractionated by SDS-PAGE and, after transfer to a polyvinylidene difluoride (PVDF) membrane, immunoreaction was carried out for $24 \mathrm{~h}$ at $4^{\circ} \mathrm{C}$ using a 1:1000 dilution of the primary antibodies. Antibodies used in immunoblotting for detecting proteins included rabbit-derived anti-actin, anti-Bcl-2, anti-Bax; goat-derived anti-cytochrome $c$ and anti-caspase-9; and anti-PARP-1 polyclonal antibodies (Santa Cruz Biotechnology, Santa Cruz, CA, USA). We also used rabbit-derived anti-caspase-3 and anti-AMPK (Cell Signaling Technology) and anti-p-AMPK (ABCAM, Cambridge, MA, USA). After reaction with horseradish peroxidase-conjugated secondary antibody, as appropriate, proteins were detected using an ECL-detecting reagent according to the manufacturer's instructions (Amersham Pharmacia Biotech, Uppsala, Sweden). The X-ray films were scanned, and the optical densities of the bands were analyzed by densitometry using the computer-based Sigma Gel program, version 1.0 (SPSS, Chicago, IL, USA).

MTT assay for cell viability. Cell viability in cultured neurons was estimated using MTT. Primary cultures of hippocampal neurons in the logarithmic growth phase were used to seed 96-well plates. Each well contained $200 \mu$ Dulbecco's modified Eagle's medium (DMEM) containing indicated supplements and $1 \times 10^{5}$ cells. The cells were incubated at $37^{\circ} \mathrm{C}$ in a humidified $5 \% \mathrm{CO}_{2}$ incubator. At the end of incubation, MTT $(5 \mathrm{mg} / \mathrm{ml}$ in PBS, $\mathrm{pH} 7.4)$ was added to each well and incubation was continued for $4 \mathrm{~h}$ at $37^{\circ} \mathrm{C}$ after which the $A_{570 \mathrm{~nm}}$, indicating viable cells, was measured using a microplate reader (Anthos 2020, Anthos Labtech Instruments, Wals, Austria). Percent cell viability was calculated as $100 \times$ the ratio of $A_{570 \mathrm{~nm}}$ of the treated cells to $A_{570 \mathrm{~nm}}$ of control cells.

Flow cytometric analysis of $\Delta \Psi \mathbf{m}$. Mitochondrial membrane potential was monitored using JC-1 mitochondrial membrane potential detection kit (Biotium Inc., Hayward, CA, USA) according to the manufacturer's protocol. Primary cultures of hippocampal neurons from G17.5 fetal brain were grown and treated with ethanol and osmotin as described earlier. After $24 \mathrm{~h}$ of drug treatment, cells in triplicate culture plates were harvested, stained with $\mathrm{JC}$ - 1 reagents at $37^{\circ} \mathrm{C}$ for $15 \mathrm{~min}$, washed twice in $1 \times$ assay buffer, and resuspended in $0.5 \mathrm{ml}$ PBS for FACS analysis (FACSCalibur Flow Cytometer; Becton Dickinson, San Jose, CA, USA). JC-1 aggregates in healthy polarized mitochondria emit red fluorescence at $590 \mathrm{~nm}$. JC-1 monomers that leak from stressed depolarized mitochondria emit green fluorescence at $530 \mathrm{~nm} .^{51}$ The red and green fluorescence was measured in the Green (FL-1) and Red (FL-2) channel of the flow cytometer, respectively. The cells were then immediately observed with a fluorescence microscope using a 'dual-band pass' filter designed to simultaneously detect fluorescein and rhodamine or fluorescein and Texas Red (Hayward, CA, USA).
Measurement of apoptosis by annexin-V staining. The AnnexinV-FLUOS Staining Kit (Roche Diagnostics GmbH, Mannheim, Germany) was used for the detection and quantification of the apoptotic cells. This kit uses a dualstaining protocol in which the apoptotic cells are stained with annexin-V (green fluorescence) and the necrotic cells are stained with PI (red fluorescence). Briefly, hippocampal neuronal cells were grown on triplicate culture plates and then treated with fresh medium (control), and fresh medium containing supplements as indicated in the figure legend for $24 \mathrm{~h}$ (Figure 7). The cells were harvested with PBS and processed for labeling with annexin-V and PI according to the manufacturer's protocol. The fluorescence was measured by flow cytometric analysis on a FACSCalibur Flow Cytometer using $488 \mathrm{~nm}$ excitation and a $515 \mathrm{~nm}$ band pass filter for fluorescein detection and a $600 \mathrm{~nm}$ filter for PI detection (FL1: Annexin-V-FLUOS; FL2: PI).

Intracellular $\mathrm{Ca}^{2+}$ measurement. The intracellular $\mathrm{Ca}^{2+}$ concentration was measured with the fluorescent $\mathrm{Ca}^{2+}$ indicator, Fura-2 acetoxymethyl ester (Fura-2AM) ${ }^{52}$ After 4 days of growth and $24 \mathrm{~h}$ of treatment, cultured cortical or hippocampal neurons (triplicate culture plates each containing $1 \times 10^{6}$ cells) were washed twice with Krebs buffer and then incubated in the DMEM media containing $5 \mu \mathrm{M}$ Fura-2AM at $37^{\circ} \mathrm{C}$ in a humidified incubator with $5 \% \mathrm{CO}_{2}$ for $60 \mathrm{~min}$. They were washed twice with Locke's solution $(\mathrm{pH} 7.8)$ and fura-2 fluorescence signals of $\left[\mathrm{Ca}^{+2}\right]_{\text {cyt }}$ were measured by a luminescence spectrophotometer (LS50B, Perkin Elmer, Boston, MA, USA). Excitation light from a Xenon lamp was alternated between 340 and $380 \mathrm{~nm}$ band-pass filters and the fluorescence emitted at $510 \mathrm{~nm}$ was revealed by a photon-counting photomultiplier. The $340 / 380-\mathrm{nm}$ fluorescence ratio, averaged over a period of $2 \mathrm{~s}$, was measured. Fluorescence signals were acquired stored and analyzed using a computer and universal imaging software, or a MicroVax II computer and software (origin 7 , Northampton, MA, USA). Intracellular calcium was determined using the ratio method as mentioned above, and from the Grynkiewicz equation. ${ }^{53}$

$$
[\mathrm{Ca}]=K_{\mathrm{d}} \times \frac{(\mathrm{R}-\mathrm{Rmin})}{(\mathrm{Rmax}-\mathrm{R})} \times \frac{\mathrm{Sf} 2}{\mathrm{Sb} 2}
$$

where $K \mathrm{~d}$ is the dissociation constant of the fura- $2 \mathrm{Ca}^{+2}$ interaction as $225 \mathrm{nM}$ in the cytosolic environment; $R$ is the fluorescence ratio at 340 and $380 \mathrm{~nm} ; R \min$ is the ratio with zero $\mathrm{Ca}^{+2} ; R \max$ is the ratio with saturating $\mathrm{Ca}^{+2}$ (using calcium chloride); $\mathrm{Sf2}$ is fluorescence at $380 \mathrm{~nm}$ with zero $\mathrm{Ca}^{+2}$; Sb2 is fluorescence at $380 \mathrm{~nm}$ with saturating $\mathrm{Ca}^{+2}$.

RNA interference and transfection. Small interfering RNAs (siRNAs) were designed and synthesized by Invitrogen (Carlsbad, CA, USA). The sequences of the sense siRNAs were: Rat AdipoR1 siRNA $5^{\prime}$-UACAACACCACUC AAGCCAAGUCCC; Rat AdipoR2 siRNA 5'-AACAGGUGUCUCUAAACUGGGC UCC; and Rat unrelated siRNA 5'-AUUUAACUUCUGGUGACGAUACUGG. Cultured primary hippocampal neuronal cells were transfected for $6 \mathrm{~h}$ with $200 \mathrm{nM}$ siRNA using Lipofectamine 2000 (Invitrogen). After the cultures were washed, the medium was replaced with DMEM containing $10 \%$ fetal bovine serum (FBS) and test compounds as indicated in the Figures. Cells were grown for $24 \mathrm{~h}$ and then analyzed.

Reverse transcriptase-PCR (RT-PCR). RT-PCR analysis was performed using cDNA from transfected and nontransfected cells as previously explained. ${ }^{54}$ Thermal cycling was performed under the following conditions: $94^{\circ} \mathrm{C}$ for $5 \mathrm{~min}, 28$ cycles at $94^{\circ} \mathrm{C}$ for $1 \mathrm{~min}, 54^{\circ} \mathrm{C}$ for $1 \mathrm{~min}$, and $72^{\circ} \mathrm{C}$ for $(1 \mathrm{~min})$, followed by $72^{\circ} \mathrm{C}(5 \mathrm{~min})$ for the final extension. The GAPDH housekeeping gene was used for normalization of target gene expression. PCR products were separated on a $1 \%$ agarose gel containing ethidium bromide and viewed under UV light. The primers used were the following: Rat AdipoR1, forward $5^{\prime}$-TCTTCCTCA TGGCTGTGATG-3', reverse 5'-AGCACTTGGGAAGTTCCTCC-3'; Rat AdipoR2, forward 5-GGAGCCATTCTCTGCCTTTC-3', reverse 5'-ACCAGATGTCACATTTG CCA-3'; GAPDH, forward 5'-GCCATCAATGACCCCTTCATT-3', reverse: 5'-CGCCTGCTTCACCACCTTCTT-3'.

Histological analysis and detection of apoptosis. FJB staining was performed as previously described. ${ }^{55}$ Briefly, immunofluorescence was performed on GD 17.5 primary hippocampal cells cultures grown in vitro on poly-D-lysinecoated chamber slides. Three-day-old cultures were treated for $24 \mathrm{~h}$ at $37^{\circ} \mathrm{C}$ as indicated in the figure legends. Treated cultures were fixed for 5 min with $4 \%$ paraformaldehyde in PBS and stored at $-70^{\circ} \mathrm{C}$. Slides were air dried for $3 \mathrm{~h}$ and 
then subjected to the following treatments in order: $10 \mathrm{~min}$ in $0.06 \%$ potassium permanganate solution, distilled water rinse, $20 \mathrm{~min}$ in $0.1 \%$ acetic acid containing $0.0004 \%$ FJB (Calbiochem, San Diego, CA, USA) and three washes in distilled water. The slides were then allowed to dry at $55^{\circ} \mathrm{C}$ for $10 \mathrm{~min}$ and viewed under a FITC filter in a confocal microscope (Olympus Fluoview FV1000, Tokyo, Japan). For PI staining, slides were dipped in PI solution $(1 \mu \mathrm{g} / \mathrm{ml})$ in PBS with gentle mixing for $20 \mathrm{~min}$ at room temperature and washed twice with PBS for $10 \mathrm{~min}$. Glass cover slips were mounted on the slides with mounting medium.

FJB and PI staining were also performed for in vivo analysis. At P7, the pups were anesthetized by intraperitoneal administration of sodium pentobarbital ( $50 \mathrm{mg} / \mathrm{g}$ body weight). Brains were removed, fixed in cold $4 \%$ neutral buffer paraformaldehyde for $48 \mathrm{~h}$, and cryoprotected by immersion into $20 \%$ sucrose phosphate buffer for $48 \mathrm{~h}$ at $4{ }^{\circ} \mathrm{C}$. Whole brains were frozen in OCT compound (Sakura, Torrance, CA, USA) and $14 \mu \mathrm{m}$ sections were made in the coronal planes (Leica cryostat CM 3050S, Nussloch, Germany). Sections were thawed mounted on the probe-on plus charged slide (Fisher, Pittsburgh, PA, USA). Tissue sections were stained with $\mathrm{FJB}$ and $\mathrm{PI}$ as described above. Images were acquired using a Zeiss fluorescent microscope (Zeiss, Gottingen, Germany) and confocal laser scanning microscope (Fluoview FV 1000, Olympus, Hamburg, Germany).

TUNNEL assay for apoptosis. In situ detection of apoptotic cell death was performed on cryosections $(16 \mu \mathrm{m})$ using the In situ Cell Death Detection kit Fluorescein, according to the manufacturer's instructions (GenScript Corporation, Piscataway, NJ, USA). Glass cover slips were mounted onto the glass slides with mounting medium. The FITC filter was used was to detect TUNEL staining (green). TUNEL-positive staining patterns were acquired with a confocal laser scanning microscope (FluoView FV1000; Olympus, Japan). TUNEL-positive cells in the different regions of each section were counted using a computer-based program.

Immunofluorescence analyses. For in situ analysis of cytochrome $c$ release and caspase- 3 expression after in vivo treatments, P7 neonatal rat pups were perfused with $4 \%$ ice-cold paraformaldehyde following $1 \times$ PBS perfusion transcardially as explained above. For hippocampal neuronal cells in primary culture, conditions of growth and treatment have been described above. Treated cultures were fixed with $4 \%$ neutral buffer paraformaldehyde and washed with cold PBS. Cytochrome $c$ was detected by incubating with mouse anti-cytochrome $c$ antibody overnight at $4^{\circ} \mathrm{C}$ and rabbit anti-mouse FITC-labeled antibody for 90 min at room temperature in dark $(1: 250$ and $1: 100$, respectively; Santa Cruz Biotechnology). Subsequently, caspase-3 expression was detected by using rabbit anti-caspase-3 antibody (Cell Signaling) and goat anti-rabbit TRITC labeled antibody (Santa Cruz Biotechnology; 1:250 and 1:100, respectively) under the same conditions. Slides were mounted with Prolong Antifade reagent (Molecular Probes, Eugene, OR, USA). Cytochrome $c$ (green) and caspase-3 (red) staining patterns were acquired using a confocal laser scanning microscope (Fluoview FV1000).

Data analysis and statistics. Images of ethidium bromide-stained gels and western blots were scanned and analyzed by densitometry using a computer based on the Sigma Gel System (SPSS). Density values were expressed as mean \pm S.E.M. Comparisons between treated groups and controls were done by Student's $t$-test to determine the significance of differences between relevant treatment groups. In every case, the acceptance level of statistical significance was $P<0.05$.

\section{Conflict of Interest}

The authors declare no conflict of interest.

Acknowledgements. This research was supported by the Pioneer Research Center Program through the National Research Foundation of Korea funded by the Ministry of Science, ICT \& Future Planning (2012-0009521) and NRF funded by Korea Government (MSIP: 2013RIA2A1A01005170).

1. Akbar M, Baick J, Calderon F, Wen Z, Kim HY. Ethanol promotes neuronal apoptosis by inhibiting phosphatidylserine accumulation. J Neurosci Res 2006; 83: 432-440.

2. Eckardt MJ, File SE, Gessa GL, Grant KA, Guerri C, Hoffman PL et al. Effects of moderate alcohol consumption on the central nervous system. Alcohol Clin Exp Res 1998; 22: $998-1040$.
3. Sokol RJ, Delaney-Black V, Nordstrom B. Fetal alcohol spectrum disorder. JAMA 2003; 290: 2996-2999.

4. Ieraci A, Herrera DG. Nicotinamide protects against ethanol-induced apoptotic neurodegeneration in the developing mouse brain. PLoS Med 2006; 3: e101.

5. Olney JW. Fetal alcohol syndrome at the cellular level. Addict Biol 2004; 9: 137-149.

6. Ikonomidou C, Bittigau P, Ishimaru MJ, Wozniak DF, Koch C, Genz K et al. Ethanol-induced apoptotic neurodegeneration and fetal alcohol syndrome. Science 2000; 287: 1056-1060.

7. Sari Y, Gozes I. Brain deficits associated with fetal alcohol exposure may be protected, in part, by peptides derived from activity-dependent neurotrophic factor and activity-dependent neuroprotective protein. Brain Res Rev 2006; 52: 107-118.

8. Sulik KK. Genesis of alcohol-induced craniofacial dysmorphism. Exp Biol Med (Maywood) 2005; 230: 366-375.

9. Montoliu C, Valles S, Renau-Piqueras J, Guerri C. Ethanol-induced oxygen radical formation and lipid peroxidation in rat brain: effect of chronic alcohol consumption. J Neurochem 1994; 63: 1855-1862.

10. Marino MD, Aksenov MY, Kelly SJ. Vitamin E protects against alcohol-induced cell loss and oxidative stress in the neonatal rat hippocampus. Int J Dev Neurosci 2004; 22: 363-377.

11. Wang ZV, Scherer PE. Adiponectin, cardiovascular function, and hypertension. Hypertension 2008; 51: 8-14.

12. Yamauchi T, Kadowaki T. Physiological and pathophysiological roles of adiponectin and adiponectin receptors in the integrated regulation of metabolic and cardiovascular diseases. Int J Obes (Lond) 2008; 32: S13-S18.

13. Goldstein BJ, Scalia RG, Ma XL. Protective vascular and myocardial effects of adiponectin. Nat Clin Pract Cardiovasc Med 2009; 6: 27-35.

14. Kubota N, Yano W, Kubota T, Yamauchi T, Itoh S, Kumagai $\mathrm{H}$ et al. Adiponectin stimulates AMP-activated protein kinase in the hypothalamus and increases food intake. Cell Metab 2007; 6: 55-68.

15. Guillod-Maximin E, Roy AF, Vacher CM, Aubourg A, Bailleux V, Lorsignol A et al. Adiponectin receptors are expressed in hypothalamus and colocalized with proopiomelanocortin and neuropeptide $Y$ in rodent arcuate neurons. J Endocrinol 2009; 200: 93-105.

16. Jeon BT, Shin HJ, Kim JB, Kim YK, Lee DH, Kim KH et al. Adiponectin protects hippocampal neurons against kainic acid-induced excitotoxicity. Brain Res Rev 2009; 61: 81-88.

17. Chen $B$, Liao WQ, Xu N, Xu H, Wen JY, Yu CA et al. Adiponectin protects against cerebral ischemia-reperfusion injury through anti-inflammatory action. Brain Res 2009; 1273: 129-137.

18. Nishimura M, Izumiya Y, Higuchi A, Shibata R, Qiu J, Kudo C et al. Adiponectin prevents cerebral ischemic injury through endothelial nitric oxide synthase dependent mechanisms. Circulation 2008; 117: 216-223.

19. Narasimhan ML, Ray B, Matilde P, Matthew A, Tesfaye M. Unexpected turns and twists in structure/function of PR-proteins that connect energy metabolism and immunity. Adv Bot Res, 2009; 51: 439-489.

20. Olney JW. New insights and new issues in developmental neurotoxicology. Neurotoxicology 2002; 23: 659-668.

21. Stanika RI, Pivovarova NB, Brantner CA, Watts CA, Winters CA, Andrews SB. Coupling diverse routes of calcium entry to mitochondrial dysfunction and glutamate excitotoxicity. Proc Natl Acad Sci USA 2009; 106: 9854-9859.

22. Szydlowska K, Tymianski M. Calcium, ischemia and excitotoxicity. Cell Calcium 2010; 47: 122-129.

23. Zhao L, Zhao ST, Qian ZM, Zhang C, Wu XM, Du F et al. Activation of group III metabotropic glutamate receptor reduces intracellular calcium in beta-amyloid peptide [31-35]-treated cortical neurons. Neurotox Res 2009; 16: 174-183.

24. Haorah J, Ramirez SH, Floreani N, Gorantla S, Morsey B, Persidsky Y. Mechanism of alcohol-induced oxidative stress and neuronal injury. Free Radic Biol Med 2008; 45: 1542-1550.

25. Marzo I, Brenner C, Kroemer G. The central role of the mitochondrial megachannel in apoptosis: evidence obtained with intact cells, isolated mitochondria, and purified protein complexes. Biomed Pharmacother 1998; 52: 248-251.

26. Jonas EA. Molecular participants in mitochondrial cell death channel formation during neuronal ischemia. Exp Neurol 2009; 218: 203-212.

27. Le DA, Wu Y, Huang Z, Matsushita K, Plesnila N, Augustinack JC et al. Caspase activation and neuroprotection in caspase-3- deficient mice after in vivo cerebral ischemia and in vitro oxygen glucose deprivation. Proc Natl Acad Sci USA 2002; 99: 15188-15193.

28. Li K, Li Y, Shelton JM, Richardson JA, Spencer E, Chen ZJ et al. Cytochrome c deficiency causes embryonic lethality and attenuates stress-induced apoptosis. Cell 2000; 101: 389-399.

29. Narasimhan ML, Coca MA, Jin J, Yamauchi T, Ito Y, Kadowaki T et al. Osmotin is a homolog of mammalian adiponectin and controls apoptosis in yeast through a homolog of mammalian adiponectin receptor. Mol Cell 2005; 17: 171-180.

30. Qi Y, Takahashi N, Hileman SM, Patel HR, Berg AH, Pajvani UB et al. Adiponectin acts in the brain to decrease body weight. Nat Med 2004; 10: 524-529.

31. Psilopanagioti A, Papadaki H, Kranioti EF, Alexandrides TK, Varakis JN. Expression of adiponectin and adiponectin receptors in human pituitary gland and brain. Neuroendocrinology 2009; 89: 38-47.

32. Miele M, Costantini S, Colonna G. Structural and functional similarities between osmotin from Nicotiana tabacum seeds and human adiponectin. PLOS One 2011; 6: 0016690. 
33. Kadowaki T, Yamauchi T. Adiponectin and adiponectin receptors. Endocr Rev 2005; 26 439-451.

34. Hopkins TA, Ouchi N, Shibata R, Walsh K. Adiponectin actions in the cardiovascular system. Cardiovasc Res 2007; 74: 11-18.

35. Shaw RJ, Lamia KA, Vasquez D, Koo SH, Bardeesy N, Depinho RA et al. The kinase LKB1 mediates glucose homeostasis in liver and therapeutic effects of metformin. Science 2005; 310: 1642-1646.

36. Green CR, Kobus SM, Ji Y, Bennett BM, Reynolds JN, Brien JF. Chronic prenatal ethanol exposure increases apoptosis in the hippocampus of the term fetal guinea pig. Neurotoxicol Teratol 2005; 27: 871-881

37. Taylor RC, Cullen SP, Martin SJ. Apoptosis: controlled demolition at the cellular level. Nat Rev Mol Cell Biol 2008; 9: 231-241.

38. Youle RJ, Strasser A. The BCL-2 protein family: opposing activities that mediate cell death Nat Rev Mol Cell Biol 2008; 9: 47-59.

39. Kretz A, Kugler S, Happold C, Bahr M, Isenmann S. Excess Bcl-XL increases the intrinsic growth potential of adult CNS neurons in vitro. Mol Cell Neurosci 2004; 26: 63-74.

40. Jung TW, Lee JY, Shim WS, Kang ES, Kim JS, Ahn CW et al. Adiponectin protects human neuroblastoma SH-SY5Y cells against MPP + -induced cytotoxicity. Biochem Biophys Res Commun 2006; 343: 564-570.

41. Correia S, Carvalho C, Santos MS, Proenca T, Nunes E, Duarte Al et al. Metformin protects the brain against the oxidative imbalance promoted by type 2 diabetes. Med Chem 2008; 4: 358-364

42. El-Mir MY, Detaille D, R-Villanueva G, Delgado-Esteban M, Guigas B, Attia S et al. Neuroprotective role of antidiabetic drug metformin against apoptotic cell death in primary cortical neurons. J Mol Neurosci 2008; 34: 77-87.

43. Tattersall DB, van Heeswijck R, Hoj PB. Identification and characterization of a fruit-specific, thaumatin-like protein that accumulates at very high levels in conjunction with the onset of sugar accumulation and berry softening in grapes. Plant Physiol 1997; 114: 759-769.

44. Barre A, Peumans WJ, Menu-Bouaouiche L, Van Damme EJ, May GD, Herrera AF et al. Purification and structural analysis of an abundant thaumatin-like protein from ripe banana fruit. Planta 2000; 211: 791-799.

45. Kritchevsky D. Caloric restriction and experimental carcinogenesis. Hybrid Hybridomics 2002; 21: 147-151.
46. Longo VD, Fontana L. Calorie restriction and cancer prevention: metabolic and molecular mechanisms. Trends Pharmacol Sci 2010; 31: 89-98.

47. Hursting SD, Smith SM, Lashinger LM, Harvey AE, Perkins SN. Calories and carcinogenesis: lessons learned from 30 years of calorie restriction research. Carcinogenesis 2010; 31: 83-89.

48. Arsenescu V, Narasimhan ML, Halide T, Bressan RA, Barisione C, Cohen DA et al. Adiponectin and plant-derived mammalian adiponectin homolog exert a protective effect in murine colitis. Dig Dis Sci 2011; 56: 2818-2832.

49. Singh NP, Lai $\mathrm{H}$, Khan $\mathrm{A}$. Ethanol-induced single-strand DNA breaks in rat brain cells. Mutat Res 1995; 345: 191-196.

50. Naseer MI, Shupeng L, Kim MO. Maternal epileptic seizure induced by pentylenetetrazol: apoptotic neurodegeneration and decreased GABAB1 receptor expression in prenatal rat brain. Mol Brain 2009; 2: 20

51. Reers M, Smith TW, Chen LB. J-aggregate formation of a carbocyanine as a quantitative fluorescent indicator of membrane potential. Biochemistry 1991; 30: 4480-4486.

52. Malgaroli A, Milani D, Meldolesi J, Pozzan T. Fura-2 measurement of cytosolic free Ca2 + in monolayers and suspensions of various types of animal cells. J Cell Biol 1987; 105: 2145-2155.

53. Grynkiewicz G, Poenie M, Tsien RY. A new generation of $\mathrm{Ca} 2+$ indicators with greatly improved fluorescence properties. J Biol Chem 1985; 260: 3440-3450.

54. Naseer MI, Lee HY, Ullah N, Ullah I, Park MS, Kim SH et al. Ethanol and PTZ effects on siRNA-mediated GABAB1 receptor: down regulation of intracellular signaling pathway in prenatal rat cortical and hippocampal neurons. Synapse 2010; 64: 181-190.

55. Schmued LC, Hopkins KJ. Fluoro-Jade B: a high affinity fluorescent marker for the localization of neuronal degeneration. Brain Res 2000; 874: 123-130.

(c) (i) (2) Cell Death and Disease is an open-access journal published by Nature Publishing Group. This work is licensed under a Creative Commons Attribution-NonCommercialShareAlike 3.0 Unported License. To view a copy of this license, visit http://creativecommons.org/licenses/by-nc-sa/3.0/

\section{Supplementary Information accompanies this paper on Cell Death and Disease website (http://www.nature.com/cddis)}

